OPEN ACCESS

Edited by:

Sergio Tombesi,

Catholic University of the Sacred

Heart, Italy

Reviewed by:

Xiaomin Yu,

Fujian Agriculture and Forestry

University, China

Yanbo $\mathrm{Hu}$,

Northeast Forestry University, China

*Correspondence:

Jianyun Ruan

jruan@mail.tricaas.com

${ }^{\dagger}$ These authors have contributed equally to this work

Specialty section

This article was submitted to

Plant Abiotic Stress,

a section of the journal

Frontiers in Plant Science

Received: 19 July 2021 Accepted: 02 September 2021

Published: 08 October 2021

Citation:

KC S, Long L, Liu M, Zhang $Q$ and

Ruan J (2021) Light Intensity Modulates the Effect of Phosphate Limitation on Carbohydrates, Amino Acids, and Catechins in Tea Plants (Camellia sinensis L.). Front. Plant Sci. 12:743781. doi: 10.3389/fpls.2021.743781

\section{Light Intensity Modulates the Effect of Phosphate Limitation on Carbohydrates, Amino Acids, and Catechins in Tea Plants (Camellia sinensis L.)}

\author{
Santosh KC ${ }^{\dagger}$, Lizhi Long ${ }^{\dagger}$, Meiya Liu, Qunfeng Zhang and Jianyun Ruan* \\ Key Laboratory of Tea Plant Biology and Resources Utilization (Ministry of Agriculture), Tea Research Institute, Chinese \\ Academy of Agricultural Sciences, Hangzhou, China
}

Metabolites are major contributors to the quality of tea that are regulated by various abiotic stresses. Light intensity and phosphorus $(\mathrm{P})$ supply affect the metabolism of tea plants. However, how these two factors interact and mediate the metabolite levels in tea plants are not fully understood. The present study investigated the consequences of different light intensity and $P$ regimes on the metabolism of carbohydrates, amino acids, and flavonoids in the Fengqing tea cultivar. The leaves and young shoots were subjected to untargeted metabolomics analysis by two-dimensional gas chromatography coupled to time-of-flight mass spectrometry (GC $\times \mathrm{GC}-\mathrm{TOF} / \mathrm{MS})$, ultra-performance liquid chromatography-quadrupole-TOF/MS (UPLC-Q-TOF/MS), and targeted analysis by high-performance liquid chromatography (HPLC) along with quantification of gene expression by quantitative real time-PCR (qRT-PCR). The results from young shoots showed that amino acids, pentose phosphate, and flavonol glycosides pathways were enhanced in response to decreasing light intensities and $\mathrm{P}$ deficiency. The expression of the genes hexokinase 1, ribose 5-phosphate isomerase A (RPIA), glutamate synthetase 1 (GS1), prolyl 4-hydroxylase (P4H), and arginase was induced by P limitation, thereafter affecting carbohydrates and amino acids metabolism, where shading modulated the responses of transcripts and corresponding metabolites caused by $P$ deficiency. $P$ deprivation repressed the expression of $\mathrm{Pi}$ transport, stress, sensing, and signaling (SPX2) and induced bidirectional sugar transporter (SWEET3) and amino acid permeases $(A A P)$ which ultimately caused an increase in the amino acids: glutamate (Glu), proline (Pro), and arginine (Arg) under shading but decreased catechins [epicatechingallate (ECG) and Gallic acid, GA] content in young shoots.

Keywords: Camellia sinensis L, phosphorus, light intensity, metabolic pathway, gene expression, interaction 


\section{BRIEF SUMMARY STATEMENT}

The interaction of light intensity and phosphorus availability at transcriptional and metabolic levels influenced amino acids, carbohydrates, and flavonoid homeostasis.

\section{INTRODUCTION}

Tea metabolites are key components of taste, flavor, and health benefits. These metabolites are largely regulated by various environmental factors such as light intensity and phosphate (Pi) availability during plant growth and development. Light is essential to photosynthesis and other subsequent physiological processes in plants. Phosphorus (P) plays an enormous role in the regulation of whole plant growth and, more importantly, the quality of tea's young shoots. Despite abundant total P in soils, the availability of $\mathrm{P}$ in tea gardens is relatively low due to the fixation with iron and aluminum in the acidic soils (Shen et al., 2011). Therefore, Pi is one of the most important fertilizers for tea productivity and quality. In agricultural practice, shading is frequently applied in the management of tea gardens as it can improve the quality of green tea by decreasing the ratio of polyphenols to amino acids and reducing the levels of galloylated catechins in tea shoots (Zhang et al., 2021). Thus, the understanding of how $\mathrm{P}$ and light affects the metabolites of tea plants, and their potential interaction will contribute to balancing the yield and quality of tea in tea production.

There is profound evidence on the effects of $\mathrm{P}$ on plant growth and the contents of other nutrients in plant organs. P deficiency negatively impacts plant growth and productivity (Stewart et al., 2001). To adapt to low P, plants may respond by modifying the root architecture and morphology (Liao et al., 2004; Zhou et al., 2008; Tian et al., 2012). Specifically, by increasing the exudation of protons and organic acids (Fox and Comerford, 1990; Ström et al., 2005; Taghipour and Jalali, 2013), enhancing the secreted or root-associated acid phosphatase activities (delPozo et al., 1999; Bozzo et al., 2002; Ligaba et al., 2004; Wang et al., 2009, 2012; Liang et al., 2010; Robinson et al., 2012), reducing the synthesis of phosphorylated metabolites (Pant et al., 2015; Ding et al., 2017), or by decreasing the evapotranspiration from leaves (Motalebifard et al., 2013). Moreover, P limitation has been reported to decrease the water-extractable concentrations of total polyphenols and free amino acids in leaves (Lin et al., 2012). In particular, the contents of amino acids such as theanine (Thea), aspartic acid (Asp), and glutamic acid (Glu) were downregulated (Lin et al., 2012; Ding et al., 2017) upon P starvation. In contrast, $\mathrm{P}$ limitation increased the concentrations of water-soluble sugars, along with the amino acids valine (Val), proline (Pro), and cysteine (Cys), and the ratio of total polyphenols to total free amino acids (Lin et al., 2012).

The elevated light intensity has been reported to increase shoot photosynthetic carbon fixation and the supply of carbohydrates to root but to decrease $\mathrm{P}$ concentration in plants (Taylor et al., 2008; Cheng et al., 2014). The leaves of tea plants cultivated in the shade contain higher amino acid contents than those grown in full sunlight due to regulation by sugar supply and a variety of regulatory signals (Lam et al., 1996). On the one hand, light regulates photosynthetic carbon assimilation (Timm et al., 2013), and shading preserves amino acids from protein synthesis (Schlüter et al., 2013), thereby reserving energy for plants during periods of extended darkness (Gipson et al., 2017). On the other hand, light regulates the expression of genes which are also involved in low $\mathrm{P}$ responses via mediating the synthesis and transport of photosynthates (Hammond and White, 2008; Zhou et al., 2020).

Numerous genes involved in sugar biosynthesis have been reported to be regulated by light and mineral availability (Lejay et al., 2008; Granot et al., 2014). For instance, the crosstalk of sugars and $\mathrm{Pi}$ is mediated by hexokinase (HXK) which could be related to downstream steps of sugar metabolism via the HXK pathway involving glycolysis rather than sugar sensing (Granot et al., 2014). Pi starvation-induced genes, such as $\beta$ $A M Y$ and $C H S$, are also sugar-induced independently from the HXK pathway, suggesting an interaction between Pi starvation signaling and HXK-independent sugar sensing in Arabidopsis (Müller et al., 2005; Ceasar, 2020). In addition, genes related to carbohydrate anabolism and catabolism (Zhang et al., 2003; Quehenberger et al., 2019), run parallel to glycolysis (Kruger and von-Schaewen, 2003), regulate ammonium assimilation (Kusano et al., 2020) and polyamine levels (Majumdar et al., 2016), and inhibit flavonol biosynthesis through polymerization (Yin et al., 2012; Wang et al., 2018), have been reported. Amino acids are responsible for tea quality. Low-quality grades of black tea were found to contain lower free amino acid contents (Alasalvar et al., 2012). In tea, the most abundant amino acids are Thea, Gln, Glu, and Arg (Ruan et al., 2012). The coefficient values in one study revealed that Gln, Arg, and Thea were the major contributors to high-ranking tea, whereas Asp and Ala were the main components in low-ranking tea (Miyauchi et al., 2014).

The biosynthesis of metabolites in the organs of tea plants responds differentially to variable light intensities and $\mathrm{P}$ supply, leading to specific changes in metabolite levels. On the one hand, the expression of genes for enzymes and transcription factors drives the synthesis of metabolites. On the other hand, the metabolites could regulate the expression and functionality of the genes by feedback loops. Thus, understanding these molecular networks on carbohydrates, amino acids, and flavonoids is a prerequisite to optimizing the effects of the related abiotic factors. Untargeted metabolomics profiling based on two dimensional gas chromatography coupled to time-of-flight mass spectrometry (GC $\times \mathrm{GC}-\mathrm{TOF} / \mathrm{MS})$ and ultra-performance liquid chromatography-quadrupole-TOF/MS (UPLC-Q-TOF/MS), integrated with targeted amino acid and catechins by high-performance liquid chromatography (HPLC) were used to investigate the effects on metabolites in mature leaves, roots, and young shoots of tea plants under different light and $\mathrm{P}$ levels. To gain a deep understanding of the molecular mechanisms under specific pathways, the expression of transcription factors and genes for metabolic biosynthesis was investigated in parallel with metabolite analysis. The objective of this study was to gain a systematic understanding of the responsive pathways, their specific metabolites, and expression of genes to light intensity, $\mathrm{P}$, and their interactional effects on tea quality components. 


\section{MATERIALS AND METHODS}

\section{Plant Materials}

The tea cultivar Fengqing was planted in pots at the Tea Research Institute, Hangzhou. The soil growth medium was prepared from thoroughly mixed pertile, vermiculite, and turf with volume in the ratio of 1:2:3 (1:2:3, v/v). Coarse sand was mixed thoroughly with the growth medium $(1: 1, w / w)$ to make it stable. The plants were exposed to full natural light (100\%; FL), medium light ( $50 \%$ of full light; ML), and low light (20\% of full light; LL) by shading with black perforated nylon agronet and measuring the light intensity in the plant vicinity using a LUX meter. The light intensity was measured weekly. The control $(+\mathrm{P})$ treatment was applied by adding $0.1 \mathrm{mM}$ of $\mathrm{P}$ as $\mathrm{KH}_{2} \mathrm{PO}_{4}$, while no $\mathrm{P}$ was added in the low $(-\mathrm{P})$ treatment. Each treatment included four pots and each pot contained four plants. Plants were irrigated with an automatic micro-sprinkler system every day to ensure an adequate supply of water.

Plants with uniform height and biomass were used for the experiment and sampling started after 1 year from stably growing plants. Young shoots (a bud with the first leaf) and leaves (fifth leaf) were harvested six times $(n=6$; three times for each in two independent experiments) during 14 weeks period. The samples were collected on the same day at the same time (9:30 am) within a range of $(10 \mathrm{~min})$ to avoid differences caused by temperature, relative humidity, and light. Materials collected for analysis were divided into two parts: one part was collected and frozen immediately in liquid nitrogen, and stored at $-80^{\circ} \mathrm{C}$, whereas the other part was dried to be used for elemental analysis. After collecting shoot samples, the remaining part of each plant was pulled out from the soil, washed carefully with de-ionized water, and the fresh fibrous root was separated, frozen in liquid nitrogen, and stored at $-80^{\circ} \mathrm{C}$ for further analysis.

\section{Processing of the Samples and Instrument Conditions for Untargeted Metabolomics}

The frozen young shoots, leaves, and roots were ground into a fine powder using liquid nitrogen. About $100 \mathrm{~g}$ of each sample was ground and exactly weighed in a $2 \mathrm{ml}$ tube under chilled conditions using ice cubicles and liquid nitrogen. For metabolomic analysis based on UPLC-Q-TOF/MS, the plant samples were extracted with $2 \mathrm{ml}$ of a solvent mixture of $75 \%$ methanol and $1 \%$ formic acid for $10 \mathrm{~min}$ in an ultrasonic bath and then centrifuged at $12,000 \mathrm{rpm}$ for $15 \mathrm{~min}$ at $-4^{\circ} \mathrm{C}$. Two milliliters of extracted samples were filtered through a $0.22 \mathrm{~mm}$ PTFE filter and injected into a 1 dram glass vessel. GC $\times$ GC-TOF/MS samples were extracted using $1,000 \mathrm{ml}$ of methanol-chloroform (3:1, v/v) solvent (Sigma-Aldrich Co., St. Louis, MO, USA) from the plant sample. Ten milliliters of L2-chlorophenyl alanine $(0.2 \mathrm{mg} / \mathrm{ml}$ in water $)$ were mixed by vortexing $(30 \mathrm{~s})$ and then centrifuged at $12,000 \mathrm{rpm}$ for $10 \mathrm{~min}$ at $-4^{\circ} \mathrm{C}$. The supernatant $(400 \mathrm{ml})$ was dried with vacuum centrifugation without heating. The samples were frozen in liquid nitrogen and $80 \mathrm{ml}$ of methoxyamine was added $(8 \mathrm{mg} / \mathrm{ml}$ in pyridine) to each dried sample followed by vortexing for $1 \mathrm{~min}$. Methoxymation and trimethylsilylation were performed as described previously (KC et al., 2018) and the glass vials were sealed for sample analysis.

The machine consisted of an Agilent GC 6890N gas chromatograph, the high-speed TOF mass spectrometer detector (Pegasus HT, Leco Co., CA, USA). It was equipped with DB-5 MS column ( $30 \mathrm{~m} \times 250 \mu \mathrm{m}$ i.d., $0.25 \mu \mathrm{m}$ film thickness) as the first dimension column and a DB-17H $(2.5 \mathrm{~m} \times 0.1 \mathrm{~mm}$ I.D., $0.1 \mu \mathrm{m}$ film thickness) as the second-dimension column. Helium was used as carrier gas at a constant flow rate of $1 \mathrm{~m} \mathrm{~min}^{-1}$. The GC oven temperature was initially set at $80^{\circ} \mathrm{C}$ for $2 \mathrm{~min}$, then raised to $180^{\circ} \mathrm{C}$ at a rate of $10-240^{\circ} \mathrm{C}$, at a rate of $5-290^{\circ} \mathrm{C}$, and finally held at $290^{\circ} \mathrm{C}$ for $9 \mathrm{~min}$. The secondary oven temperature was kept at $5^{\circ} \mathrm{C}$ offset above the primary oven. Each $1 \mu$ l aliquot of the derivatized sample was injected in splitless mode, and the injector temperature was set at $270^{\circ} \mathrm{C}$. The temperatures of the transfer line and ion source were set at 260 and $200^{\circ} \mathrm{C}$, respectively. The mass spectrometry data were acquired with electron impact ionization $(70 \mathrm{eV})$ at full scan mode $(\mathrm{m} / \mathrm{z} 30-600)$. The dwelling time for each scan was set at a rate of 20 spectra per second and the solvent delay at $3 \mathrm{~min}$.

The metabolites were achieved in the ultra-performance liquid chromatography (ACQUITY UPLC, Waters Corp., Milford, MA), which was equipped with an Acquity HSS T 3 column $\left(1.8 \mathrm{~mm}, 100 \mathrm{~mm}, 62.1 \mathrm{~mm}\right.$; Milford, MA, USA) kept at $40^{\circ} \mathrm{C}$, and connected to a quadrupole-time of flight mass spectrometer (Xevo G2-XS QTOF, Waters Corp.). Mobile phase A was $0.1 \%$ aqueous formic acid, and mobile phase B was acetonitrile containing $0.1 \%$ formic acid. Gradient elution with a flow rate of $0.4 \mathrm{ml} / \mathrm{min}$ was performed as follows: the gradient profile was $4 \% \mathrm{~B}$ from 0 to $1 \mathrm{~min}, 4-7 \% \mathrm{~B}$ at $1-3 \mathrm{~min}, 7-25 \% \mathrm{~B}$ at $3-5 \mathrm{~min}, 25-45 \% \mathrm{~B}$ at $5-8 \mathrm{~min}, 45-90 \% \mathrm{~B}$ at $8-10 \mathrm{~min}, 90-$ $100 \% \mathrm{~B}$ at $10-12 \mathrm{~min}$, and $100 \% \mathrm{~B}$ at $12-15 \mathrm{~min}$. The injection volume of the samples was $2 \mu \mathrm{l}$, and the samples were kept at $6^{\circ} \mathrm{C}$ during analysis. After each injection, the needle was rinsed with $600 \mu \mathrm{l}$ of weak wash solution (water/methanol, 90:10) and $200 \mu \mathrm{l}$ of strong wash solution (methanol/water, 90:10). Mass spectra were acquired using electrospray ionization at positive and negative modes over the range of $\mathrm{m} / \mathrm{z} 100-170$. The stability of the method was tested by performing 10 repeated injections of the mixed samples every $2 \mathrm{~h}$. The maximal tolerated $\mathrm{m} / \mathrm{z}$ deviation, minimum or maximum chromatographic peak width in consecutive scans, and allowable retention time deviations were set as $15 \mathrm{ppm}, 5 / 20 \mathrm{~s}$, and $2 \mathrm{~s}$, respectively.

\section{Quantifying Targeted Catechin and Amino Acids by HPLC}

Samples for catechin determination were treated as described for UPLC-Q-TOF/MS (KC et al., 2018). For free amino acid analysis, samples were derivatized as recommended by the waters AccQ Tag chemistry package. HPLC analysis was carried out using an e2695 connecting 2,998 photodiode array detector system (Waters Corp., Milford, MA, USA) injected with 10 or $25 \mu \mathrm{l}$ of sample solutions for catechin or free amino acid, respectively. For catechins, distilled water with $2 \%$ formic acid was used as mobile phase A. Mobile phase B consisted of 
HPLC solvent Acetonitrile (ACN). The samples were eluted at column temperature $40 \pm 1^{\circ} \mathrm{C}$ at a flow rate of $1 \mathrm{ml} / \mathrm{min}$ and monitored at $278 \mathrm{~nm}$. Similarly, for amino acids, the AccQTag eluent from waters was used as mobile phase A. Mobile phase $\mathrm{B}$ was $\mathrm{ACN}$, and the column temperature was set at $37 \pm 2^{\circ} \mathrm{C}$. The procedure was completed as prescribed in the AccQ Tag chemistry package instruction manual. Peaks of catechins were identified by comparing the retention times of the samples to those of authentic standards and amino acids were identified as prescribed in the manual.

\section{qRT-PCR and ICP AES Analysis}

The extracted RNA was used to synthesize cDNA using PrimeScript Reagent Kit. Transcripts of each gene were quantified as cp value using Light Cycler 480 II real-time machine with Light Cycler 480 Software version 4.0 (Roche, Mannheim, Germany; Singh et al., 2015). To integrate the corresponding changes, four different $\mathrm{P}$ responsive genes and 19 metabolic genes from different pathways were selected and used to manifest the response to $\mathrm{P}$ treatments under different light intensities. The primer sequences of the genes used for qRT-PCR are shown in Supplementary Tables 1, 2 and the reference gene GAPDH with sample $(\mathrm{FL}+\mathrm{P})$ was used for normalization (Xiong et al., 2013). The $P$ concentrations in freeze-dried plant samples were measured by ICP-AES following digestion with concentrated $\mathrm{HNO}_{3}$ and $\mathrm{HClO}_{4}$ (Salahinejad and Aflaki, 2010).

\section{Data Processing, Analysis, and Visualization}

The data were collected from two independent experiments with three biological replicates each. The whole metabolomics data were screened and identification was performed prior to normalizing the data. Data files from GC $\times$ GC-TOF/MS were processed in Leco software (Leco Co., St. Joseph, MI, USA) and were deconvoluted using Automatic Mass spectral Deconvolution and Identification Software (AMDIS). The corresponding peaks of each chromatogram were compared to the National Institute of Standards and Technology (NIST, FairCom Co., Gaithersburg, MD, USA) mass spectral database to identify metabolites. Raw chromatographic data acquired from the UPLC-Q-TOF/MS analysis were processed by TransOmics (Waters Corp., Milford, MA, USA), and peaks were annotated from the accurate mass measurements using online metabolite databases. The metabolites were identified based on actual mass, retention time, and isotopic distribution, and accurate mass measurements were confirmed from Metlin online web-based database and our published literature (KC et al., 2018).

After normalization (a relative expression to FL+P), PCA analysis was performed using the R package "MetaboAnalystR" on samples from a different light and $\mathrm{P}$ treatments to observe for PLS-DA test for separation of the group (Supplementary Figure 1) and data analysis for the enriched pathways and pathway impact. Similarly, the data from HPLC and ICP-AES were used to measure the contents of targeted metabolites and nutrition content in young shoots and leaves under different light and $\mathrm{P}$ treatments. Furthermore, all the spectrometric measurements were analyzed with two-way
ANOVA to compare means of single light effect, $\mathrm{P}$ effect and their interaction, and one-way ANOVA to compare means of full light (FL), medium light (ML), low light (LL), and shading effect $(\mathrm{FL} \times \mathrm{ML}$ and $\mathrm{LL})$ using Tukey's HSD with $\mathrm{R}$ package "agricolae." The enriched pathways, pathway impact, pathway map, bar plot, box plot, and circular plot were drawn using the latex “tkiz" package. Pearson's correlations were performed using the R statistical package "pcorr." The linear regression graph and bi-plot PCA figure and heatmap figure were drawn collectively using the $\mathrm{R}$ and Latex program.

\section{RESULTS}

\section{Plant Biomass and Element Concentrations}

The dry weights of all tissues were significantly decreased under $\mathrm{P}$ deficiency in ML (50\% of full light) conditions (Table 1; Supplementary Figure 2). The shading treatment increased the total biomass of young shoots, leaves, and roots in ML compared to $\mathrm{FL}$ and $\mathrm{LL}$ with $\mathrm{P}$ sufficient conditions whereas the total biomass in LL was decreased compared to FL and ML under Pdeficient conditions. In contrast, both 50\% (ML) and 80\% (LL) shading treatments did not significantly affect the total biomass of young shoots, leaves, and roots under $\mathrm{P}$ deficiency. Thus, the interaction between light regimes and $\mathrm{P}$ levels was observed in terms of the biomass of young shoots and leaves. The $\mathrm{P}$ concentrations in young shoots and leaves decreased under $\mathrm{P}$ deficiency regardless of the light intensity. Upon decreasing the light intensities from FL to LL, P concentration in young shoots was reduced under both P-sufficient and P-deficient conditions (Table 1; Supplementary Figure 3).

The shading treatments also affected the concentrations of other elements in P-sufficient and P-deficient plants (Table 2; Supplementary Figure 3 ). In young shoots, the concentrations of $\mathrm{K}, \mathrm{Mg}, \mathrm{S}, \mathrm{Ca}, \mathrm{Cu}, \mathrm{Fe}, \mathrm{B}$, and $\mathrm{Al}$ decreased but those of $\mathrm{Mn}$ and $\mathrm{Zn}$ increased under shading. On the other hand, $\mathrm{P}$ limitation decreased the concentrations of $\mathrm{Mg}, \mathrm{Ca}, \mathrm{Cu}, \mathrm{Zn}, \mathrm{Mn}$, and $\mathrm{Al}$ in young shoots under FL but increased $\mathrm{S}$ concentration regardless of light intensity. Similarly, S concentration decreased in young shoots under shading but increased in leaves under $\mathrm{P}$ limitation regardless of the light intensity. Taken together, the concentrations of elements, except $\mathrm{Mn}$ and $\mathrm{Zn}$, decreased under shading and further decreased due to $\mathrm{P}$ deficiency in young shoots and leaves (Table 2; Supplementary Figure 3).

\section{Comparative Effects of Different Light and P Levels on Untargeted Metabolites Young Shoots}

The metabolite sets enrichment ratio (MSEA) and pathway impact (PI) in young shoots were influenced by light intensities and $\mathrm{P}$ supply (Figure 1). The carbohydrate related pathways including pentose phosphate pathway $(\mathrm{MSEA}=8.14$; $\mathrm{PI}=0.37)$, inositol phosphate metabolism (MSEA $=3.08$; PI $=0.13$ ), and fructose and mannose metabolism (MSEA $=1.18$; PI $=0.22$ ) were affected. A similar impact was noticed on amino acid pathways, viz., phenylalanine metabolism $(\mathrm{MSEA}=2.21$; $\mathrm{PI}=$ 
TABLE 1 | Biomass and concentrations of phosphorus ( $P$ ) in the organs of Fengqing cultivar under different light intensities and phosphorous (P) levels.

\begin{tabular}{|c|c|c|c|c|c|c|c|c|}
\hline Parameter & Organ & P level & \multicolumn{3}{|c|}{ Light intensity } & \multicolumn{3}{|c|}{ Significance } \\
\hline \multirow{6}{*}{$\begin{array}{l}\text { Biomass } \\
\left(\text { g plant }^{-1}\right)\end{array}$} & & $-\mathrm{P}$ & $3.48 \pm 0.48 c$ & $4.1 \pm 0 . .31 c$ & $5 \pm 0.78 b c$ & & & \\
\hline & Leaves & $+\mathrm{P}$ & $4.72 \pm 0.89 a b$ & $6.06 \pm 0.61 a$ & $3.86 \pm 0.93 b$ & $p<0.01$ & $p<0.001$ & $p<0.01$ \\
\hline & & $-\mathrm{P}$ & $3.71 \pm 0.48 b$ & $3.88 \pm 0.98 b$ & $3.97 \pm 0.85 b$ & & & \\
\hline & & $-P$ & $9.97 \pm 1.4 a b$ & $9.07 \pm 2.42 b$ & $5.43 \pm 0.55 c$ & & & \\
\hline & Total & $+\mathrm{P}$ & $21.68 \pm 1.97 b$ & $26.66 \pm 3.75 a$ & $17.68 \pm 1.26 b c$ & $p<0.001$ & $p<0.001$ & $p<0.01$ \\
\hline & & $-P$ & $17.16 \pm 1.42 c$ & $17.04 \pm 3.08 c$ & $14.41 \pm 1.34 c$ & & & \\
\hline \multirow[t]{3}{*}{$\mathrm{P}\left(\mathrm{mg} \mathrm{g}^{-1}\right)$} & Young Shoots & $+\mathrm{P}$ & $9.57 \pm 0.09 a$ & $6.52 \pm 0.15 c$ & $4.76 \pm 0.06 d$ & $p<0.001$ & $p<0.001$ & $p<0.001$ \\
\hline & & $-P$ & $7.62 \pm 0.04 b$ & $4.24 \pm 0.08 e$ & $2.49 \pm 0.02 f$ & & & \\
\hline & Leaves & $+\mathrm{P}$ & $9.08 \pm 0.34 a$ & $4.2 \pm 0.22 d$ & $5.1 \pm 0.2 c$ & $p<0.001$ & $p<0.001$ & $p<0.001$ \\
\hline
\end{tabular}

Means with different letters in the same row of the same parameter and organ are significantly different. ns, non-significant differences, between light and $P$ interaction.

TABLE 2 | Concentrations of macronutrients $\left(\mathrm{K}, \mathrm{Mg}, \mathrm{Ca}, \mathrm{S}\right.$, and $\left.\mathrm{Al}, \mathrm{mg} \mathrm{g}^{-1}\right)$ and micronutrients $\left(\mathrm{Zn}, \mathrm{Mn}, \mathrm{Cu}, \mathrm{Fe}, \mathrm{B}, \mathrm{mg} \mathrm{kg}^{-1}\right)$ in young shoots under different light intensities and P levels.

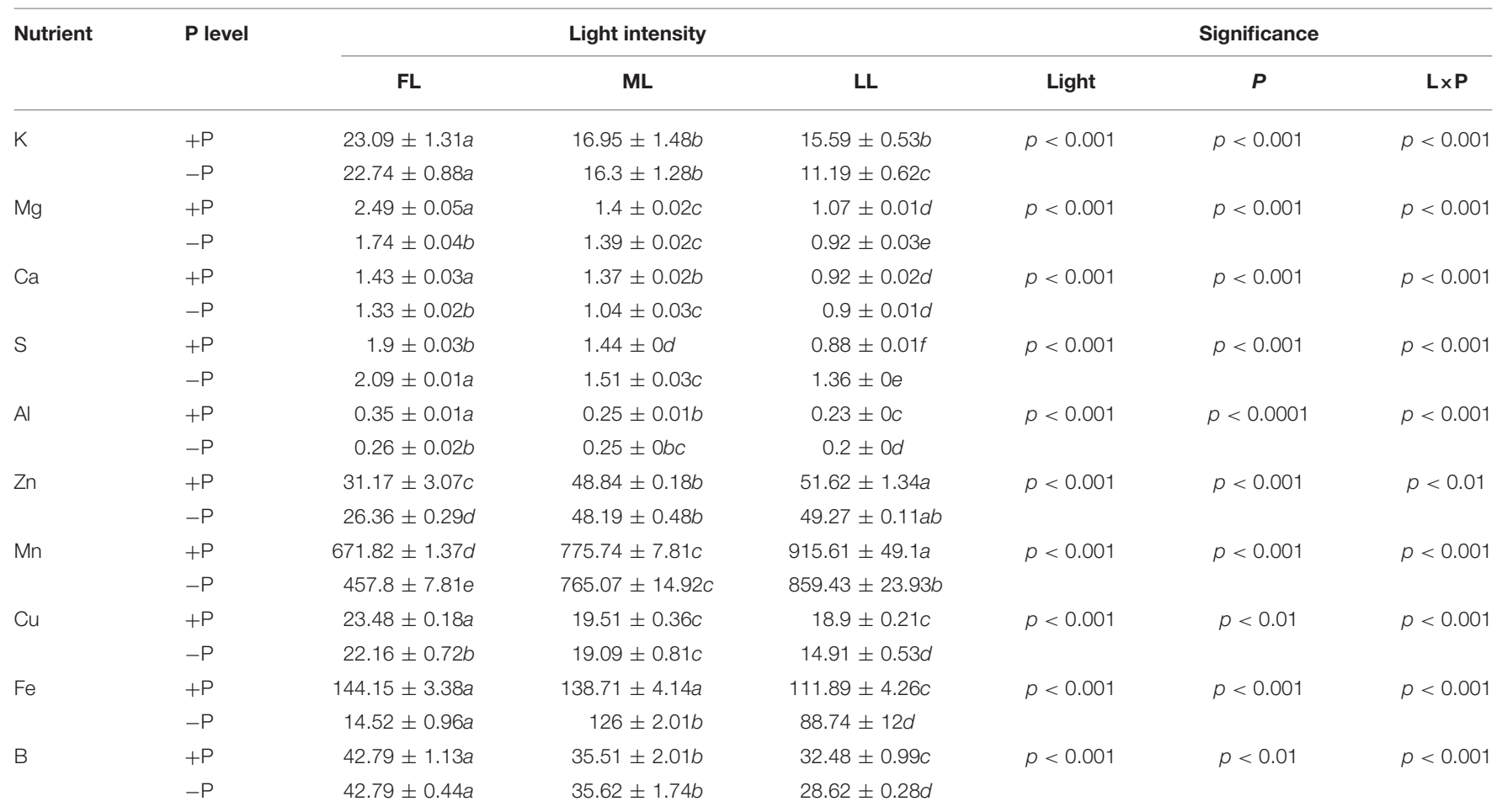

Means with different letters in the same row of the same parameter and organ are significantly different. ns, non-significant differences, between light and $P$ interaction.

0.47), TCA cycle (MSEA $=6.09 ; \mathrm{PI}=0.29)$, alanine, aspartate, and glutamate metabolism (MSEA $=3.7 ; \mathrm{PI}=0.72)$, and arginine and proline metabolism (MSEA $=8.98$; PI $=0.40)$. Similarly, pathways related to secondary metabolites including flavone and flavonol biosynthesis $(\mathrm{PI}=0.7)$ and flavonoid biosynthesis $(\mathrm{PI}=$ $0.46)$ were also greatly influenced by light intensity and P levels.

In Figure 2 and Supplementary Table 3, the untargeted metabolites with respect to carbohydrates or anaplerotic pathways were detected in young shoots in response to different light intensities and $\mathrm{P}$ supply. In young shoots, within the fructose and mannose metabolism, D-mannose increased remarkably by $\mathrm{P}$ limitation from 1 to $13.4(p<0.001)$ in FL, and from 0.3 to $2.38(p<0.001)$ in LL, whereas mannose-6P decreased by $-\mathrm{P}$ treatment from 1 to $0.61(p<0.001)$ and from 0.97 to $0.28(p<0.001)$ under FL and LL conditions, respectively. Fructose and mannose metabolism related metabolites were 
A

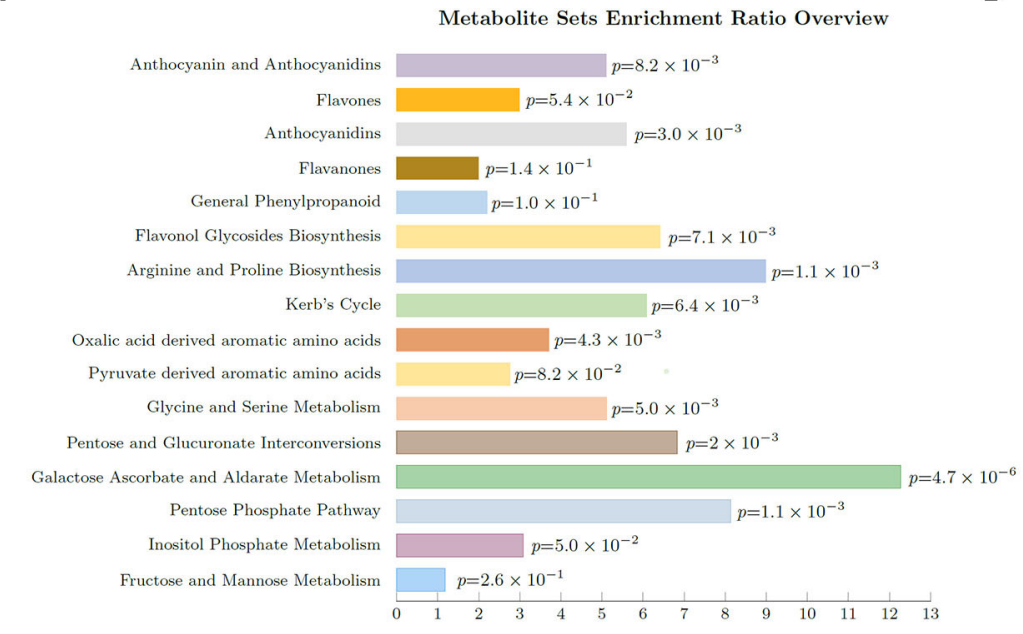

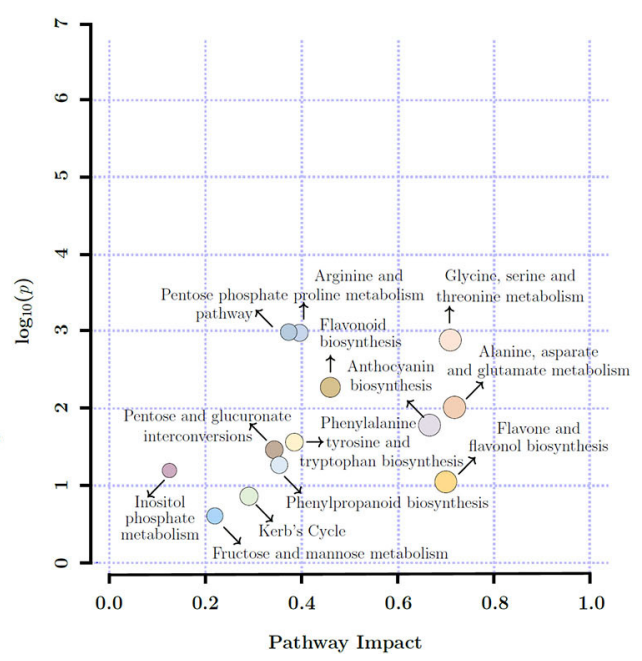

FIGURE 1 | Overview of enriched pathways (A) and pathway impact (B) in young shoots of Fengqing cultivar exposed to different light intensities and Phosphorous (P) levels.

responsive to sole light, $\mathrm{P}$, and their interaction effect. In inositol phosphate metabolism pathway, myoinositol-1P was reduced by $\mathrm{P}$ limitation from 1 to $0.76(p<0.01)$ in FL and 1.11 to $0.44(p<0.001)$ in LL. By contrast, Myo-Inositol was increased from 1 to $2.76(p<0.001)$ by P supply under LL and raised from 1 to $8.81(p<0.001)$ by P deficiency under FL condition. In the pentose phosphate pathway, there was an opposite effect of shading on gluconate under P sufficiency and deficiency. On the other hand, $\mathrm{P}$ limitation increased gluconate from 1 to $8.57(p<0.001)$ in FL, whereas it decreased gluconate from 7.85 to $1.84(p<0.001)$ in ML and from 7.82 to 1.79 ( $p$ $<0.001)$ in LL. D-ribulose-5P increased significantly by P limitation regardless of light intensities. P deficiency increased D-ribose $-5 \mathrm{P}$ but decreased ribose under FL and LL conditions. In addition, the light intensities affected the metabolic changes induced by $\mathrm{P}$ limitation in galactose ascorbate and aldarate metabolism (Figure 2). The interaction between light intensity and $\mathrm{P}$ supply was also observed in pentose and glucuronate interconversions (Supplementary Table 3).

In glycine and threonine metabolism, there was a mean increase in L-serine (from 1 to 2.24; $p<0.001$ ), glycine (from 1 to $2.24 ; p<0.001$ ), threonine (from 1 to $4.19 ; p<0.001$ ), and $O$-phospho-L-homoserine (from 1 to $1.44 ; p<0.001$ ) by $\mathrm{P}$ deficiency in FL (Figure 3; Supplementary Table 3). In the same pathway, there was no decrease in $O$-phospho-L-homoserine (from 0.19 to $0.18 ; p>0.005$ ) under LL condition. The pyruvatederived amino acids, $\mathrm{L}$-isoleucine (from 0.21 to $0.08 ; p<0.001$ ) and L-valine (from 0.48 to $0.36 ; p<0.001$ ) decreased due to $\mathrm{P}$ limitation. L-tyrsone significantly increased (from 1 to 33.58) by LL in P-sufficient condition but decreased (from 33.58 to $0.94)$ by P deficiency under LL $(p<0.001)$. In alanine, asparate, and glutamate metabolism, L-alanine (from 0.59 to $0.22 ; p<$ 0.001 ), L-asparagine (from 0.89 to $0.2 ; p<0.001$ ), and asparate 4-semialdehye (from 1.62 to $0.84 ; p<0.001$ ) decreased due to $\mathrm{P}$ limitation. There was a decrease in $\mathrm{L}$-asparagine (from 0.27 to $0.2 ; p<0.001$ ) and increase in asparate 4 -semialdehyde (from 1.62 to $7.27 ; p<0.01$ ) under shading condition. Lglutamate increased (from 1.68 to $5.69 ; p<0.001$ ) due to $\mathrm{P}$ limitation under all light conditions, but L-glutamate (from 0.29 to $0.36 ; p<0.001)$ showed only mild increase under LL at $\mathrm{P}$ condition. Oxalic acid (from 0.5 to 2.34; $p<0.001$ ), citrate (from 0.84 to $1.16 ; p<0.001$ ), and isocitrate (from 0.87 to 1.25 ; $p<0.001)$ increased under LL at $-\mathrm{P}$ condition in Kerb's cycle. Similarly, succinate (from 0.56 to $0.11 ; p<0.001$ ) decreased while fumarate (from 1.34 to 1.77; $p<0.001$ ) increased in comparison between $\mathrm{P}$ deficiency and $\mathrm{P}$ supply under all light treatments. In arginine and proline biosynthesis pathways, under shading condition, L-proline (from 0.87 to $16.87 ; p>0.05$ ) insignificantly increased whereas 4 -hydroxyproline (from 0.74 to $0.31 ; p<0.001)$ significantly decreased in comparison between $\mathrm{P}$ deficiency and $\mathrm{P}$ supply. In arginine and proline biosynthesis pathways with all light treatments, L-arginine (from 1.36 to 1.88 ; $p<0.05$ ) increased but ornithine (from 0.74 to $0.42 ; p<0.001$ ) decreased in comparison between $P$ deficiency and $P$ supply.

Figure 4 and Supplementary Table 4 show the influence of untargeted flavonoids with respect to light intensity and P supply in young shoots. There was a mean increase in quercertin (from 1 to $2.25 ; p<0.001$ ), isoquercertin (from 1 to 2.34; $p<0.001$ ), and rutin (from 1 to 3.11; $p<0.001$ ) in FL at $-\mathrm{P}$ but isoquerertin decreased (from 0.25 to $0.14 ; p<$ 0.001 ) due to shading conditions. Caffeoylshikimic acid (from 1.63 to 7.18$)$ increased due to P deficiency $(p<0.001)$ and shading $(p<0.001)$. In flavanones biosynthesis pathways, there was an opposite response of narigenin and eriodictyol to $\mathrm{P}$ effect, where narigenin decreased while eriodictyol increased or vice versa. The anthocyanidin biosynthesis metabolite, 


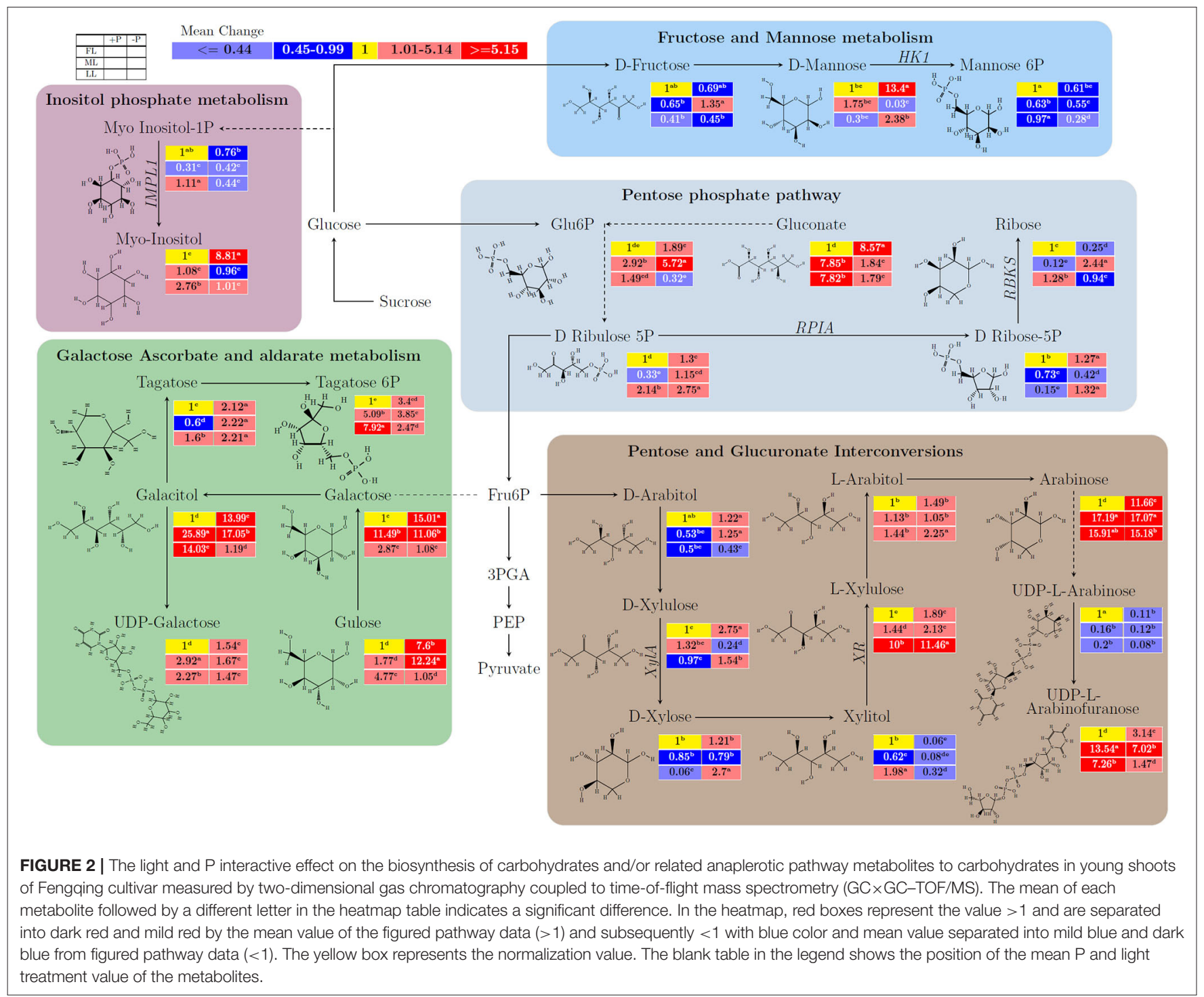

leucodelphinidin (from 0.64 to $0.33 ; p<0.001$ ) decreased due to P limitation. Similarly, delphinidin (from 0.96 to 0.23 ; $p<$ 0.001 ) decreased due to shading. There was a consistent decrease in the flavones biosynthesis-related metabolites kaempferol (from 0.31 to $0.04 ; p<0.001$ ) and malvidin (from 0.76 to $0.02 ; p<0.001)$ due to shading. The $\mathrm{P}$ limitation decreased kaempferol (from 0.87 to $0.03 ; p<0.001$ ) and luteolin (from 1.32 to $0.07 ; p<0.001$ ) regardless of light intensity. In anthocyanidin and anthocyanidins biosynthesis pathways, leucocyanidin decreased (from 0.05 to 0.02 ; $p<$ 0.001 ), and cyanidin increased (from 0.35 to $2.65 ; p<$ 0.001 ) due to P-deficient conditions in comparison between $\mathrm{P}$ deficiency and $\mathrm{P}$ supply under all light treatments. There was a decrease in leucocyanidin (from 0.08 to $0.02 ; p<0.001$ ) and cyanidin 3 -glucoside (4.7 to 2.93; $p>0.05$ ) whereas cyanidin (from 0.03 to $0.35 ; p<0.05$ ) and procyanidin B1 (from 0.23 to $0.36 ; p<0.05$ ) increased under LL at P-deficient conditions.

\section{Leaves}

In leaves, Myo inositol-1P increased (from 1.14 to 1.54; $p<$ 0.01 ) and Myo-inositol decreased insignificantly (from 0.97 to $0.66 ; p>0.05$ ) under ML due to P limitation. D-ribulose (from 1 to $0.51 ; p<0.001$ ), D-ribose-5P (from 1 to 0.96 ; $p<0.001$ ), and ribose (from 1 to $0.58 ; p<0.001$ ) decreased under FL at $-\mathrm{P}$ condition (Supplementary Table 3 ). Low light increased D-ribulose (from 0.55 to $0.62 ; p<0.001$ ), D-ribose-5P (from 0.18 to $1.31 ; p<0.001$ ) but decreased ribose (from 2.46 to $1.27 ; p<0.001)$ in comparison between $\mathrm{P}$ deficiency and $\mathrm{P}$ supply. Limitation of $\mathrm{P}$ increased D-xylulose (from 2.23 to 7.14; $p<0.001$ ) and D-xylose (from 1.58 to $4.04 ; p<0.001$ ) but decreased xylitol (from 2.23 to $0.11 ; p<0.001$ ) and L-xylulose (from 3.16 to $0.88 ; p<0.05$ ) under FL condition. ML decreased D-xylulose (from 7.14 to 2.23; $p<0.001$ ), D-xylose (from 4.04 to $1.05 ; p<0.01$ ), and xylitol (2.23 to $1.67 ; p<0.001)$ but increased L-xylulose (1.61 to 3.16; $p<0.001$ ) compared with other light treatments due to $\mathrm{P}$ limitation. L-glutamate 


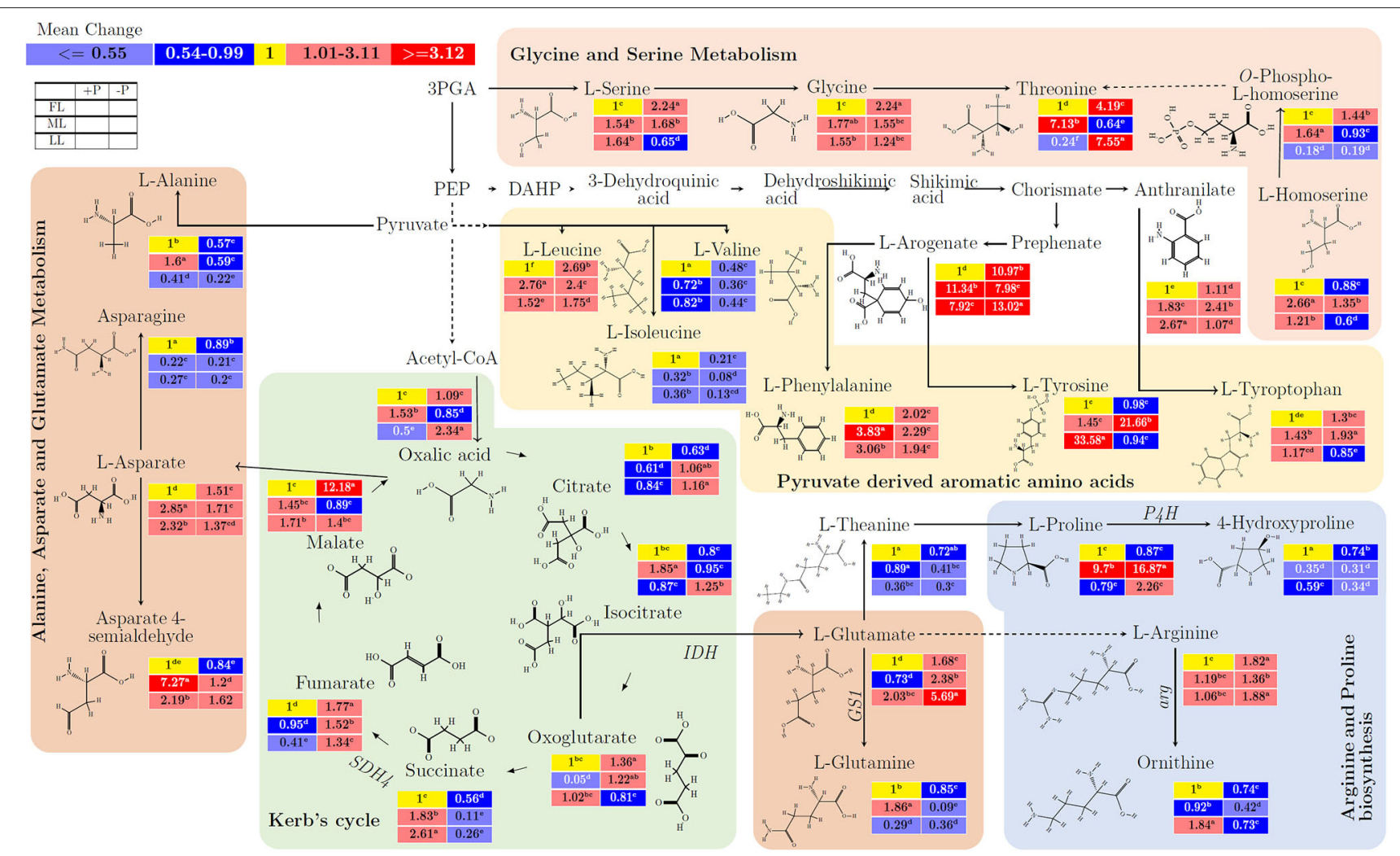

FIGURE 3 | The interactive effect of light and $\mathrm{P}$ on the biosynthesis of amino acids and/or related anaplerotic pathway metabolites in young shoots of Fengqing cultivar measured by GC $\times$ GC-TOF/MS. The mean of each metabolite followed by a different letter in the heatmap table indicates a significant change in light and $P$ treatments. In the heatmap, red boxes represent the value $>1$ and are separated into dark red and mild red by the mean value of the figured pathway data $(>1)$ and subsequently $<1$ with blue and mean value separated into mild blue and dark blue from figured pathway data $(<1)$. The yellow box represents the normalization value. The blank table in the legend shows the position of the mean $\mathrm{P}$ and light treatment value of the metabolites.

(from 0.7 to 0.35 ) and L-glutamine (from 0.76 to 0.2 ) decreased significantly in FL and ML. However, they increased under LL due to P-deficient conditions. Under shading, succinate (from 0.43 to $1.47 ; p<0.001$ ) and fumaric acid (from 1.21 to 1.49 ; $p<0.001)$ increased in ML but decreased significantly in LL due to P limitation. L-proline (from 1.51 to $0.68 ; p<0.001$ ) and hydroxyproline (from 4.44 to $1.57 ; p<0.001$ ) decreased in LL due to P limitation. Shading increased quercetin (from 0.52 to $0.62 ; p<0.001$ ) but decreased isoquercitrin (from 1.48 to $1.42 ; p>0.05$ ) and rutin (from 1.52 to $1.19 ; p<0.01$ ) due to P limitation (Supplementary Table 4). Leucodelphinidin (from 1 to $1.18 ; p<0.001$ ) and delphinidin (from 1 to 5.66 ; $p<$ 0.001 ) increased but myricetin (from 1 to 0.45 ; $p<0.001$ ), (+)gallocatechin (from 1 to $0.21 ; p<0.001$ ), and delphinidin $3-$ glucoside (from 1 to $0.68 ; p<0.001$ ) decreased under FL at $-\mathrm{P}$ condition. In ML, (+)-gallocatechin (from 0.4 to $0.54 ; p<$ 0.001 ) and delphinidin (from 3.17 to 4.6 ; $p<0.001$ ) increased, but myricetin (from 0.14 to $0.82 ; p<0.001$ ), leucodelphinidin (from 0.93 to $0.84 ; p<0.01$ ), and delphinidin 3-glucoside (from 0.99 to $0.76 ; p<0.001$ ) decreased in comparison between $\mathrm{P}$ deficiency and $\mathrm{P}$ supply under FL and LL. Leucocyanidin (from 1 to 5.92; $p<0.001$ ) increased in FL but decreased (from 5.54 to $3.07 ; p<0.01$ ) under shading compared to other light intensities under P limitation. Cyanidin (from 2.64 to 4.1 ) increased significantly but cyanidin 3-glucoside (from 0.86 to 0.1 ) decreased significantly under P limitation compared to other light intensities.

\section{Overview of Targeted Metabolomics Analysis of Tea Plants Under P-Deficient and P-Sufficient Conditions Young Shoots}

The free amino acids and flavonoids contents were significantly altered by light and $\mathrm{P}$ treatment, and hence, were grouped based on light effect, $\mathrm{P}$ effect, or light-P interaction. The loading treatments from PCA directly link to correlation analysis for group separation (Figures 5A,B; Supplementary Table 5). In young shoots, $\mathrm{P}$ was grouped with Ser and GC that had high contribution on principal components (Figure 5A). The Ser content decreased in FL $(0.79,-\mathrm{P} /+\mathrm{P})$ and $\mathrm{LL}(0.75)$ in young shoots but it increased in $\mathrm{ML}$ (1.17) due to $\mathrm{P}$ limitation (Figure 5C). Similarly, shading decreased Ile (0.65) and Val (0.68) at P-deprivation (Figures 5C-E). Glu (1.17), Pro (1.22), and Arg (2.31) increased under shading due to $\mathrm{P}$ limitation (Figures 5F-H). GC (1.15) increased in LL, whereas ECG (0.77) and GA (0.94) decreased under shading due to $\mathrm{P}$ limitation (Figures 5I-K). 


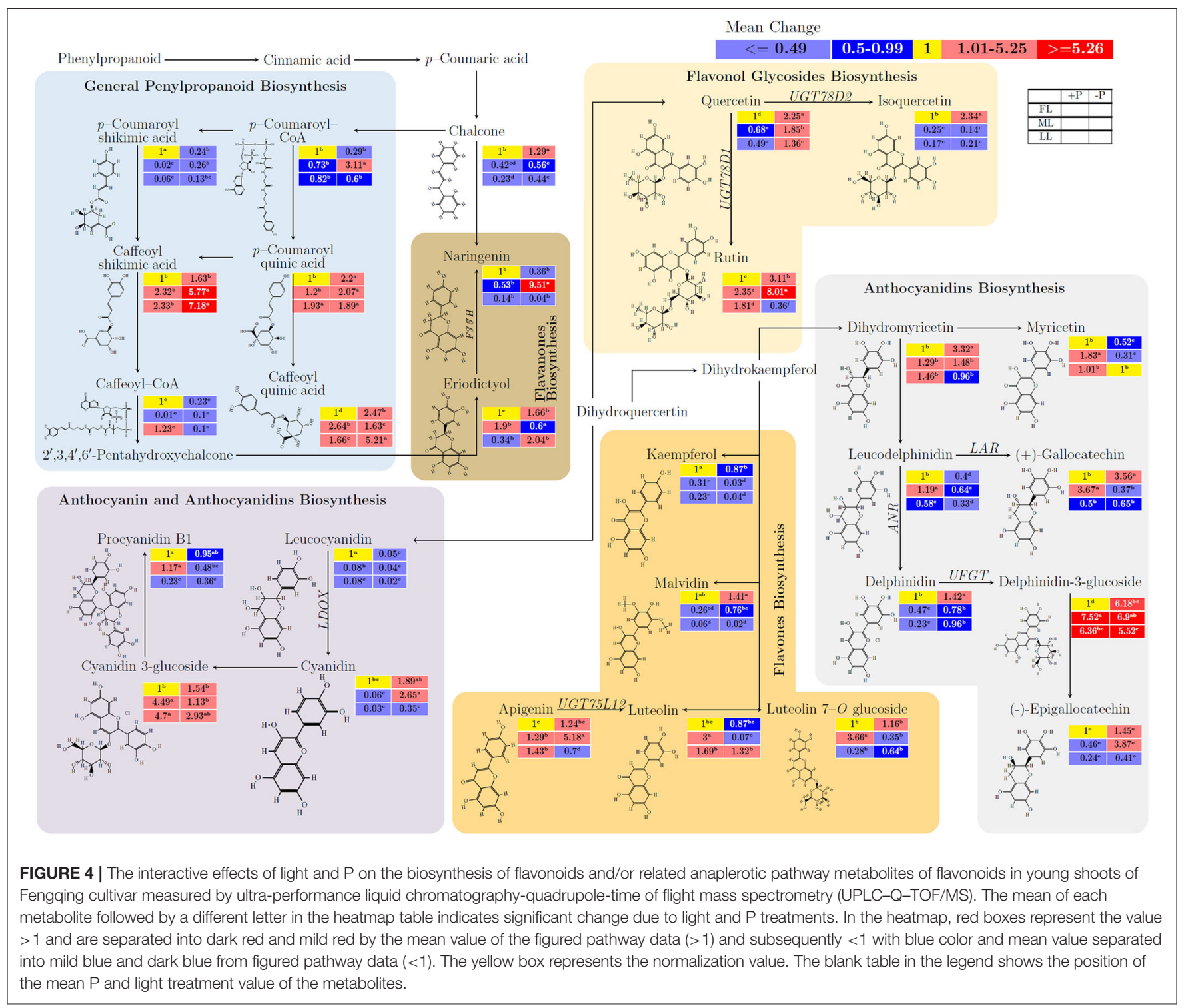

\section{Leaves}

In leaves, $\mathrm{P}$ was grouped with Ser and Asp with a high contribution on principal components (Figure 5B). P deficiency decreased the content of Ser in FL (0.67) and ML (0.53), however, there was a mild increase in LL (1.33) with the comparison between $\mathrm{P}$ deficiency and $\mathrm{P}$ supply under all light treatments (Supplementary Table 5). Ile (1.06) and Phe (1.13) increased in FL but shading decreased Ile (0.19), Val (0.5), and Phe (0.49) contents due to $\mathrm{P}$ limitation. Full light conditions decreased Asp (0.9) and Glu (0.87) under P limitation. Shading decreased Asp (0.45) but increased Glu (1.83) due to P limitation. Arg (3.74) increased in all light intensities at -P conditions. EGC (1.53) and GC (1.29) increased in FL due to P limitation. Shading increased EGC (1.61) but GC (0.58) was decreased in LL at $-\mathrm{P}$ conditions.

\section{Relative Expression of Genes in Response to $P$ Limitation and Light Changes}

The expression of the transcription factor Pi transport, stress, sensing, and signaling (SPX2) was upregulated by $\mathrm{P}$ supply under each light treatment (Figures 6, 7). Shading decreased SPX2 expression level when $\mathrm{P}$ was limited. In addition, bidirectional sugar transporter (SWEET3) was upregulated due to P limitation while it was downregulated under shading conditions. P limitation increased the transcripts of the amino acid transporter gene amino acid permeases (AAP) under all the light treatments and it was also increased by shading treatments. The interactive effect of light and $\mathrm{P}$ on the expression levels of SWEET 3 and $A A P$ were not significant while it was significant on the transcript level of glutathione S-transferase b (GSTb). There was an upregulation response of GSTb) by P limitation regardless 
Young Shoots

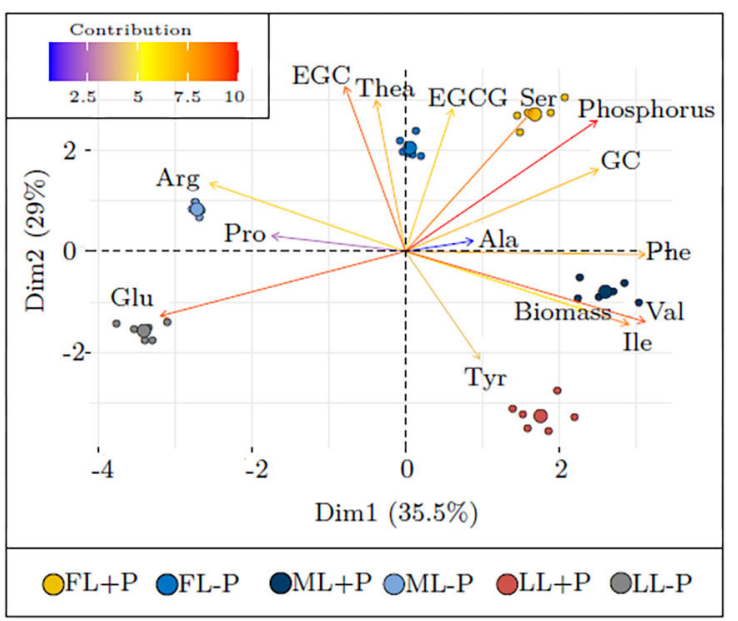

c

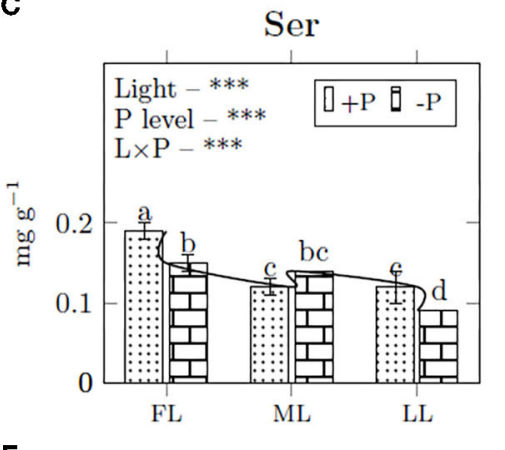

$\mathbf{F}$

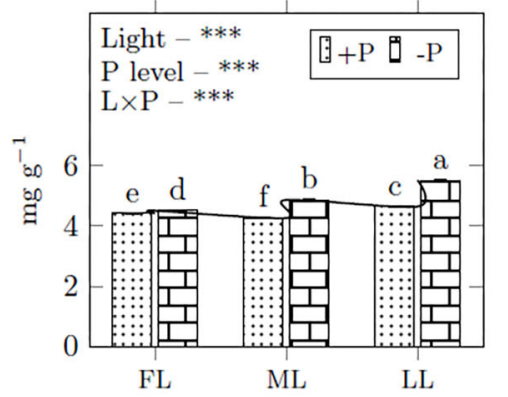

I

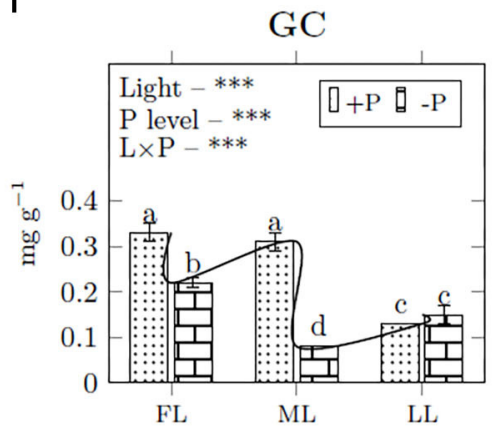

D

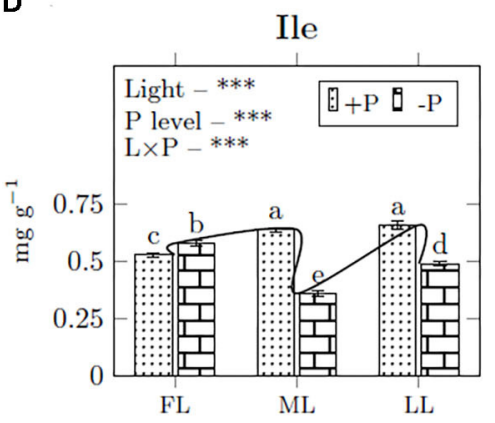

G

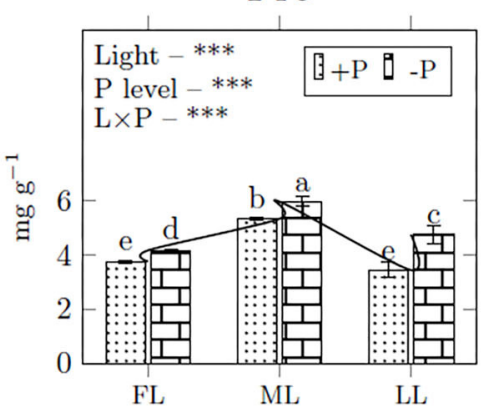

ECG

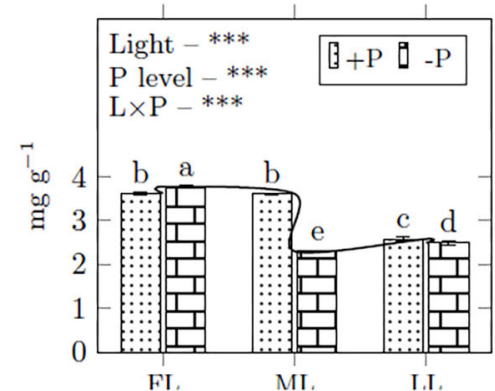

Leaves

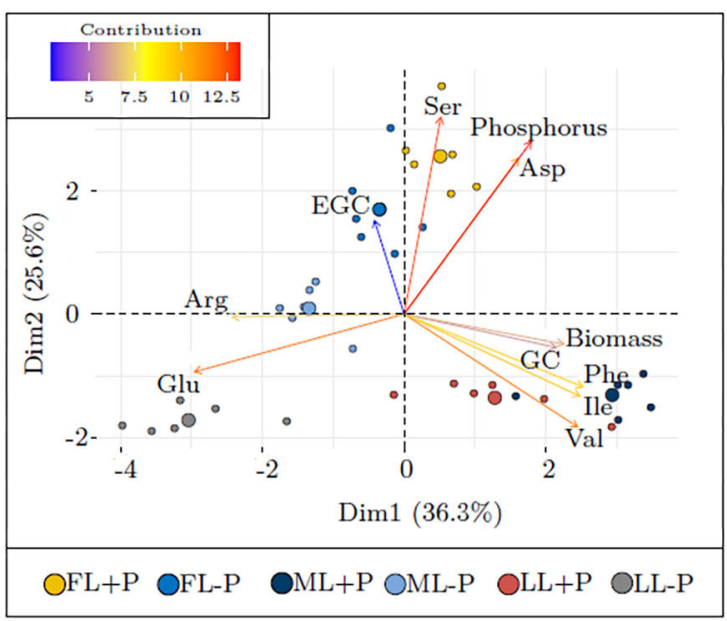

E

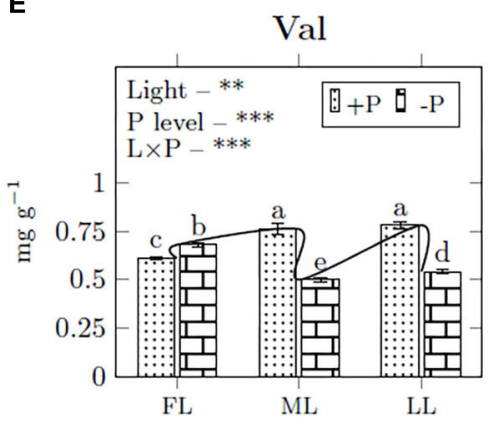

H

Arg

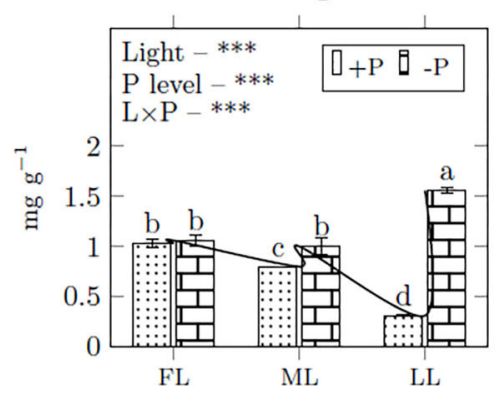

GA

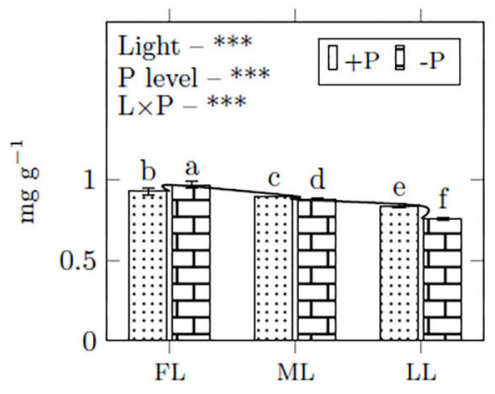

FIGURE $\mathbf{5}$ | The bi-plot correlation of young shoots (A) and leaves (B) and the mean concentration of amino acids (C-I) and catechins (J,K) in young shoots. A single bi-plot for the dataset combines both samples and treatments to the principal components; Dim1, dimension 1, and Dim2, dimension 2. Asterisks indicate significant differences: ${ }^{\star \star \star}$ is $0.001,{ }^{\star \star}$ is 0.01 , and ${ }^{\star}$ is 0.05 , ns, non-significant differences, between light and $\mathrm{P}$ interaction. 
of the light treatments. The expression of most metabolic genes investigated was upregulated in young shoots due to $\mathrm{P}$ limitation (Supplementary Figures 4-6) but a change in arg was not statistically significant. No significant interaction between light and $\mathrm{P}$ was found on the expression of ribose 5-phosphate isomerase A (RPIA), arg, and prolyl-4-hydroxylase $(P 4 H)$. Ribokinase synthase, inositol-phosphate phosphatase (IMPL1), xylose isomerase (XylA), isocitrate dehydrogenase $(I D H)$, leucoanthocyanidin dioxygenase (LDOX); however, anthocyanidin 3-O-glucosyltransferase (UFGT) was upregulated in LL due to P limitation. Shading upregulated $I D H$ and UFGT metabolic genes. With increasing $\mathrm{P}$ concentration, the expression levels of SWEET3, AAP, and GSTb were highly induced.

\section{Heatmap Correlation Clustering of Metabolites and Gene Expression Under P-Deficient and P-Sufficient Conditions}

Figure 8A shows the heat map of correlation between biosynthetic pathway metabolites with the selected genes under P-sufficient conditions. Flavonol glycosides and flavones biosynthesis related genes, UGT78D1, UGT78D2, and UGT75L12, were clustered into the same hierarchal group to show similar expression patterns of correlations with all discussed pathways in FL at P-sufficient conditions (Figure 8A). In addition, SWEET3, AAP, SPX2, carbohydrate, and amino acid biosynthesis and/or related anaplerotic pathways related genes were clustered into the same hierarchal group to show similar expression levels of correlations in FL at P-sufficient conditions. Interestingly, GSTb was clustered with all secondary metabolic genes to show its distinct and similar expression of correlations in FL at P-sufficient conditions. Similarly, in ML at P-sufficient conditions, SPX2, SWEET3, and AAP were also clustered into the same hierarchal group and GSTb showed a different cluster pattern with metabolic genes. Likewise, in LL at P-sufficient conditions, SPX2, SWEET3, and AAP were also clustered into the same hierarchal group and GSTb showed a different hierarchal cluster pattern with metabolic genes. Notably under FL and P-deficient conditions, SPX2 and GSTb formed a different cluster group while SWEET3 and AAP were also clustered into the same hierarchal group to show a similar expression of correlations with metabolic genes (Figure 8B). Similarly, SPX2, AAP, and SWEET3 were clustered into the same group with metabolic genes in ML and LL at P-deficient conditions.

\section{Overview of Metabolic Pathways and Involved Genes}

Amino acid permeases and SWEET3 were positively correlated under different light and $\mathrm{P}$ regimes. Similarly, both $A A P$ and SWEET3 were positively correlated with flavonol glycosides biosynthesis in FL at P-sufficient conditions (Figure 9). Similarly, the cluster of fructose and mannose related metabolites in FL at P-deficient conditions was strongly correlated with pentose phosphate pathway at ML and flavonol glycosides, and flavones biosynthesis at LL due to $\mathrm{P}$ deficiency. In addition, under shading conditions, flavonol glycosides biosynthesis was positively correlated with inositol phosphate metabolism. There was a strong negative correlation between FL-P of $A A P$ and $\mathrm{ML}+\mathrm{P}$ of flavonol glycosides biosynthesis. Fructose and mannose metabolism (FMM) under P deficiency at FL conditions was negatively correlated with flavonol glycosides biosynthesis under ML conditions. FMM that was due to ML under P deficiency was negatively correlated with flavones biosynthesis under LL conditions. Flavones and flavonol glycosides metabolites under shading were negatively correlated with the cluster of pentose phosphate pathway metabolites under shading. Most of the clustered inositol phosphate metabolites that were due to $\mathrm{P}$ limitation were negatively correlated with flavones biosynthesis metabolites under shading conditions (Figure 9).

\section{DISCUSSION}

The interaction between light intensity and $\mathrm{P}$ has been widely reported in plants, such as soybean (Wang et al., 2020), maize (Zhou et al., 2020), and white lupin (Cheng et al., 2014). Previous studies have focused on the influence of light intensity on low$\mathrm{P}$ induced root morphology and $\mathrm{Pi}$ acquisition. Nevertheless, how light intensity affects the P-related metabolic processes is yet to be understood, especially in the xylophyta tea plants which are sensitive to light changes. In the present study, the interaction between light intensity and $\mathrm{P}$ nutrition was identified in the shoot of tea plants and this interaction affected the carbohydrates, amino acids, flavonoids, and related anaplerotic pathways significantly.

In Fructose and Mannose metabolism, D-fructose, a monosaccharide, is transformed into mannose and further metabolized by HXK into mannose-6P. It has been reported that black tea contains high levels of Fructose (Stilo et al., 2020). The present study showed that either ML or P limitation at FL decreased D-fructose where it was metabolized into D-mannose in the young shoots (Figure 2). The decrease in D-fructose was further intensified under $80 \%$ shading combined with $\mathrm{P}$ limitation by upregulating the expression of $H X K$, which produces the substrate, mannose-6P. This is consistent with its role as a regulator for the crosstalk of sugars and $\mathrm{Pi}$ in Arabidopsis where HXKs were reported to be able to sense the sugar level and coordinate mineral uptake by the root (Lejay et al., 2008; Granot et al., 2014). In Inositol phosphate metabolism, Inositol-phosphate phosphatase 1 (EC 3.1.3.25) produces the substrate Myo-inositol which acts as a precursor with various roles in plant signal transduction and environmental responses (Gillaspy, 2011). In LL, both Myo-inositol-1P and Myo-inositol contents declined due to P deficiency (Figure 2). By contrast, the upregulated expression of IMPL1 induced by $\mathrm{P}$ limitation decreased Myo-inositol-1P and increased Myoinositol under full light conditions (Supplementary Figure 4; Figure 2), suggesting different signaling pathways in response to $\mathrm{P}$ deficiency at different light intensities. In addition, Dribose-5P could result from phosphorylation of ribose by $R B K S$ (EC 2.7.1.15) or synthesized from D-ribulose $5 \mathrm{P}$ by RPIA (EC 5.3.1.6). These processes play essential roles in carbohydrate anabolism and catabolism, for instance, in the pentose phosphate pathway that runs parallel to glycolysis 
Pi transport, stress, sensing and signalling (SPX2)

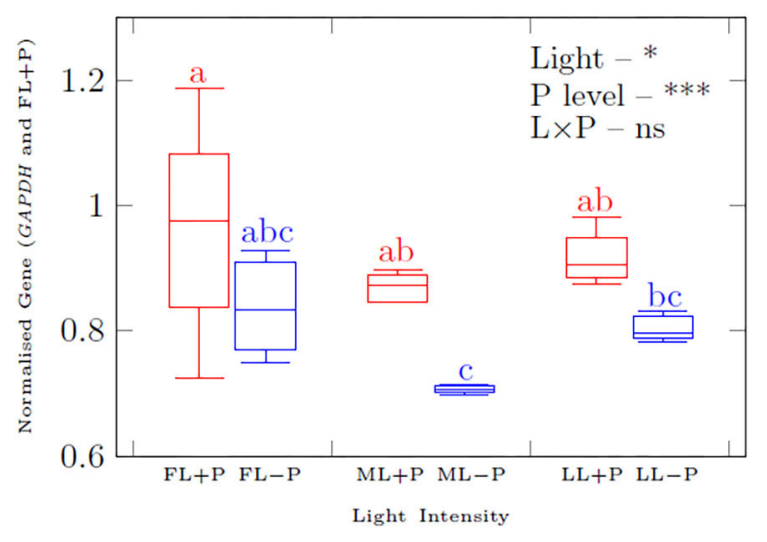

amino acid permeases $(A A P)$

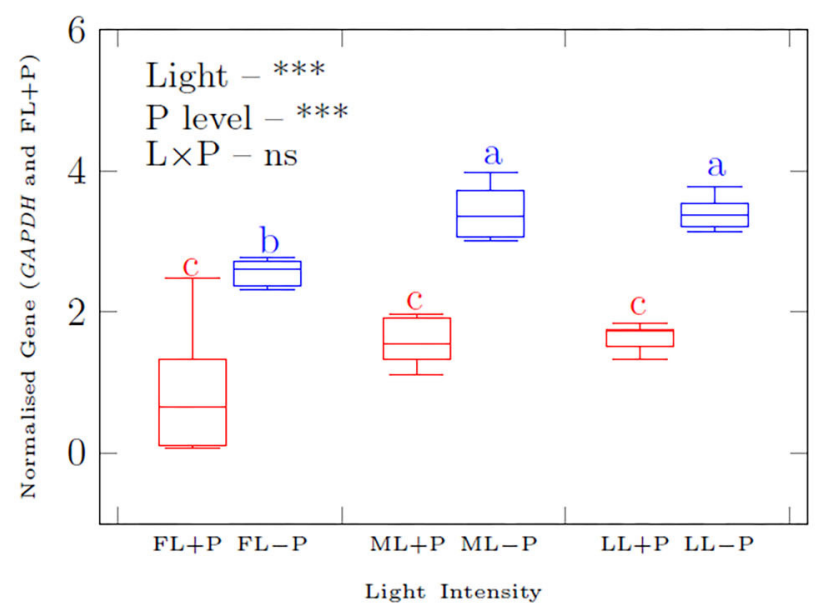

bidirectional sugar transporter (SWEET3)

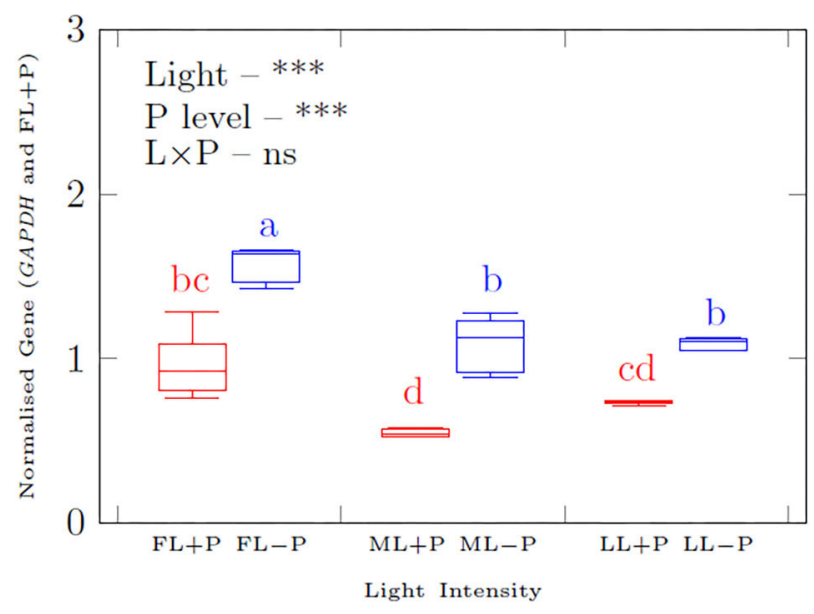

glutathione S-transferase b (GSTb)

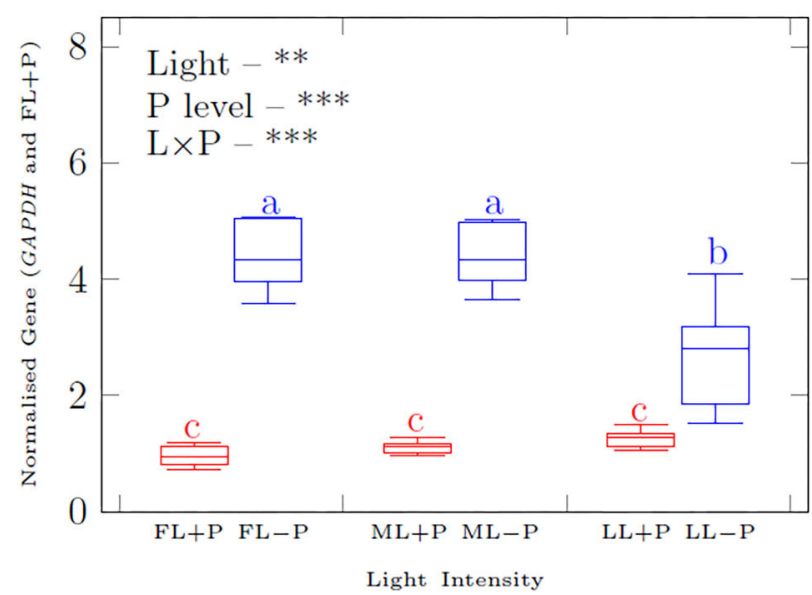

FIGURE 6 | qRT-PCR analyzed (normalized with reference gene delta CT) transporter gene transcripts normalized relative expression in response to full light with +P. Asterisks indicate significant differences: ${ }^{* *}$ is $0.001,{ }^{* \star}$ is 0.01 , and ${ }^{*}$ is $0.05, \mathrm{~ns}$, non-significant differences, between light and P interaction.

(Kruger and von-Schaewen, 2003; Zhang et al., 2003). Under the FL and LL conditions, the induced expression of RPIA and RBKS by $\mathrm{P}$ limitation resulted in the accumulation of $\mathrm{D}$-Ribose-5P (Figure 2; Supplementary Table 3, Supplementary Figure 4). Whereas D-ribose-5P decreased and ribose increased due to $\mathrm{P}$ limitation under $\mathrm{ML}$ although the expression of $R B K S$ was induced, indicating differential regulation of the pathway in ML compared to FL and LL. In ML, ribose is a crucial source for $\mathrm{NADPH}$ generation due to $\mathrm{P}$ deficiency for reductive biosynthesis. Likewise, xylose isomerase (EC 5.3.1.5) catalyzes the interconversion of D-xylose to D-xylulose. The enzyme Dxylose reductase (EC 1.1.1.307) belongs to the aldose reductase family and catalyzes the reduction of D-xylose to the sugar alcohol xylitol (Quehenberger et al., 2019). The response of Dxylulose and L-xylulose is likely related to the opposite relation on carbon utilization. This causes L-xylulose partition on ethanol production which tends to the growth of young shoots despite shading and $\mathrm{P}$ limitation.

In the targeted metabolite analysis, the concentration of serine declined under shading in young shoots due to a reduction in the photosynthetic carbon assimilation (Timm et al., 2013). In contrast to FL and LL, an increase in serine by P limitation was detected in ML which was also reported previously in the Pdeficient maize leaves (Schlüter et al., 2013). Shading treatment increased Ile and Val under P-sufficient conditions. This was a response that has also been reported previously for Arabidopsis (Gipson et al., 2017), presumably to preserve these essential amino acids for protein synthesis as an adaptive mechanism in young shoots. In general, most amino acids have been reported to present at higher levels in the shaded tea leaves (Yang et al., 2012). In the present study, phenylalanine was remarkably increased by half-light intensity compared with full light intensity when 
A

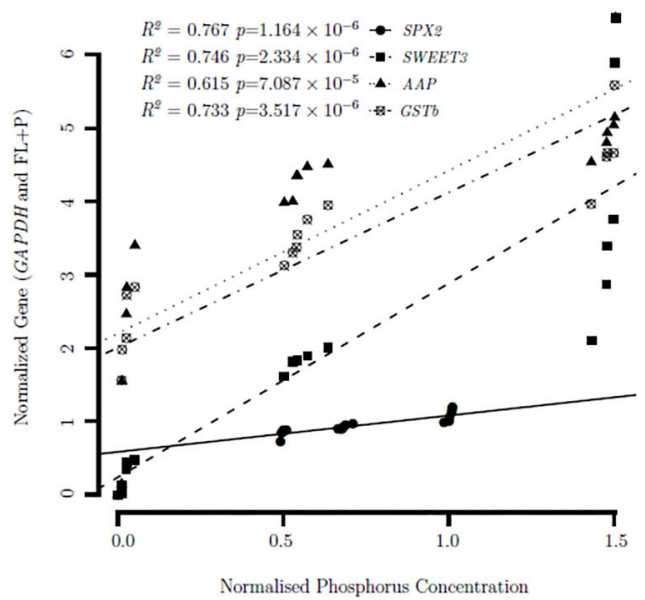

c $\mathrm{FL}$

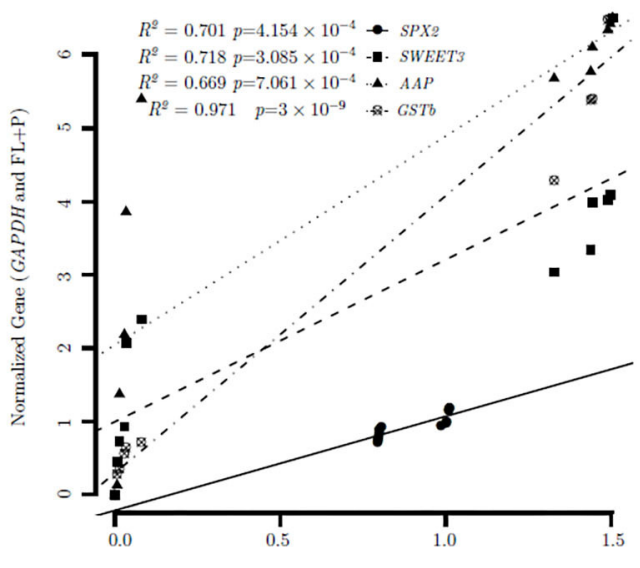

Normalised Phosphorus Concentration

E LL

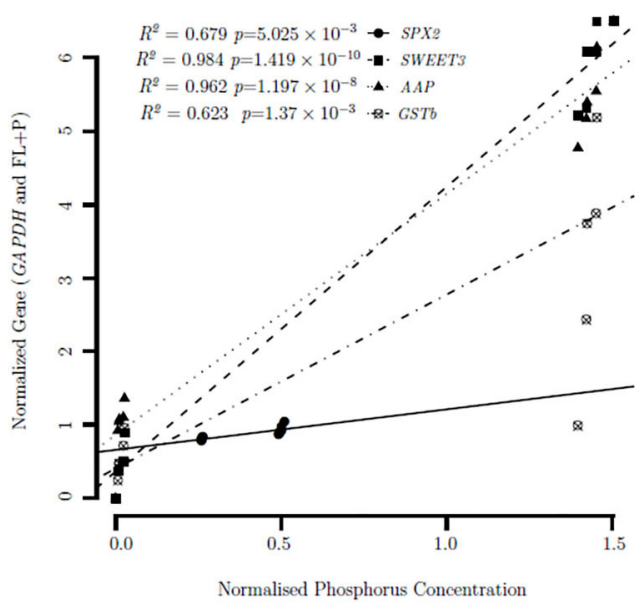

B

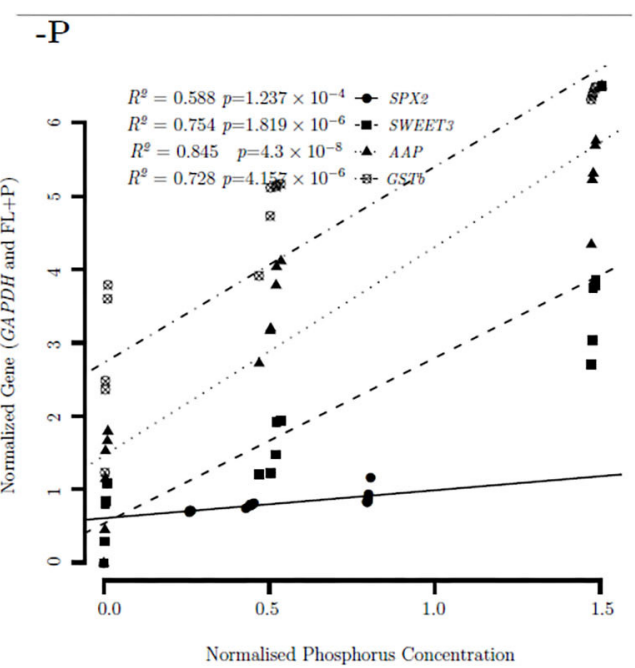

D ML

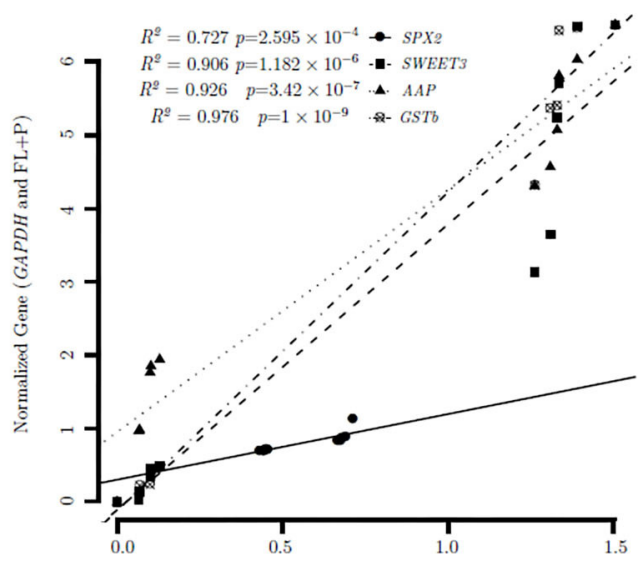

Normalised Phosphorus Concentration

FIGURE 7 | Linear regression correlation between gene expression under different P levels (A,B) and light regimes (C-E). High P (A), low P (B), full light (FL) (C), medium light (ML) (D), and low light (LL) (E) correlation of gene expression under different $P$ levels and light intensities of Pi transport, stress, and signaling (SPX2), bidirectional sugar transporter 3 (SWEET3), amino acid permeases (AAP), and glutathione S-transferase b (GSTb) in young shoots. 
A

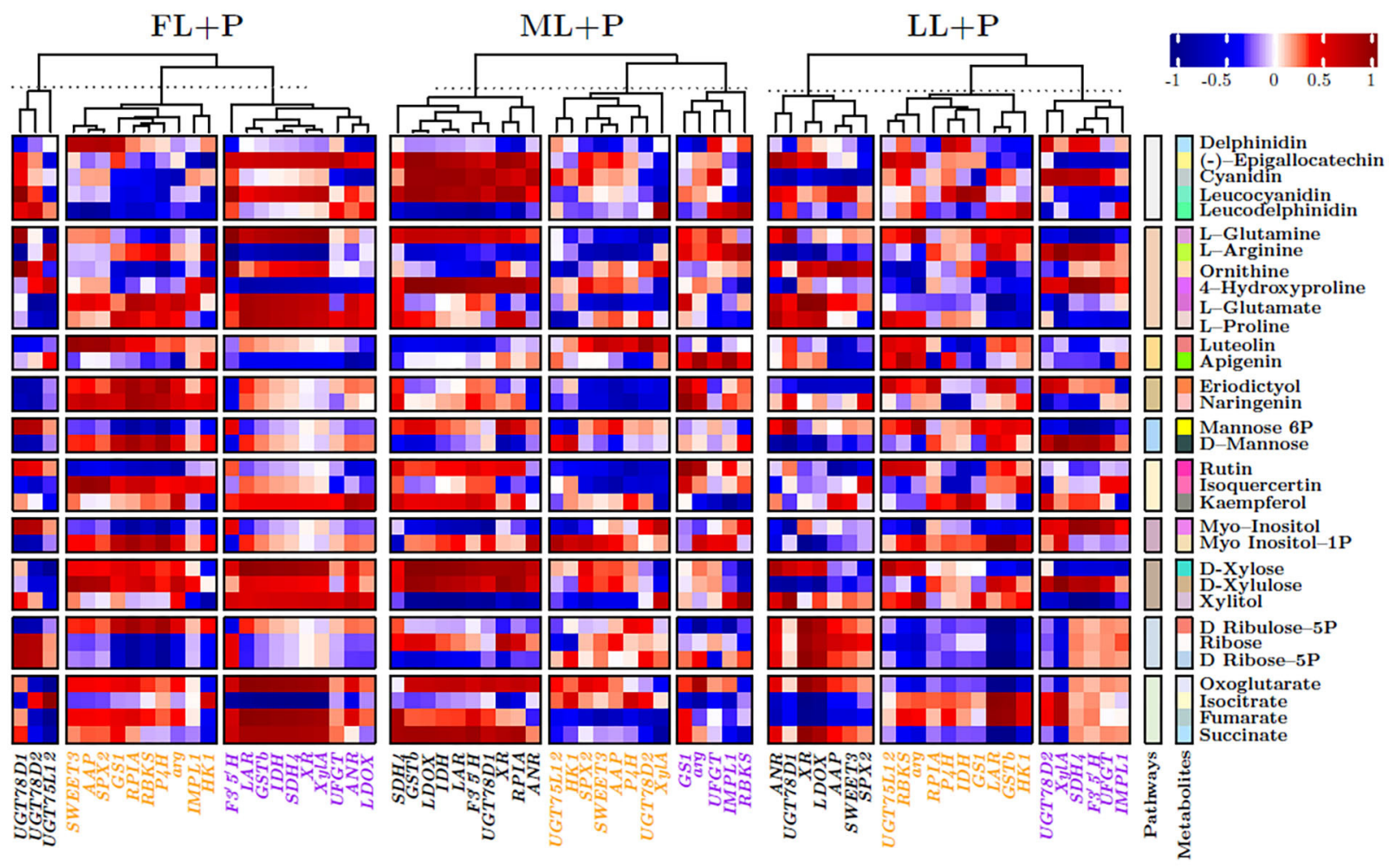

B

FL-P

ML-P

LL-P
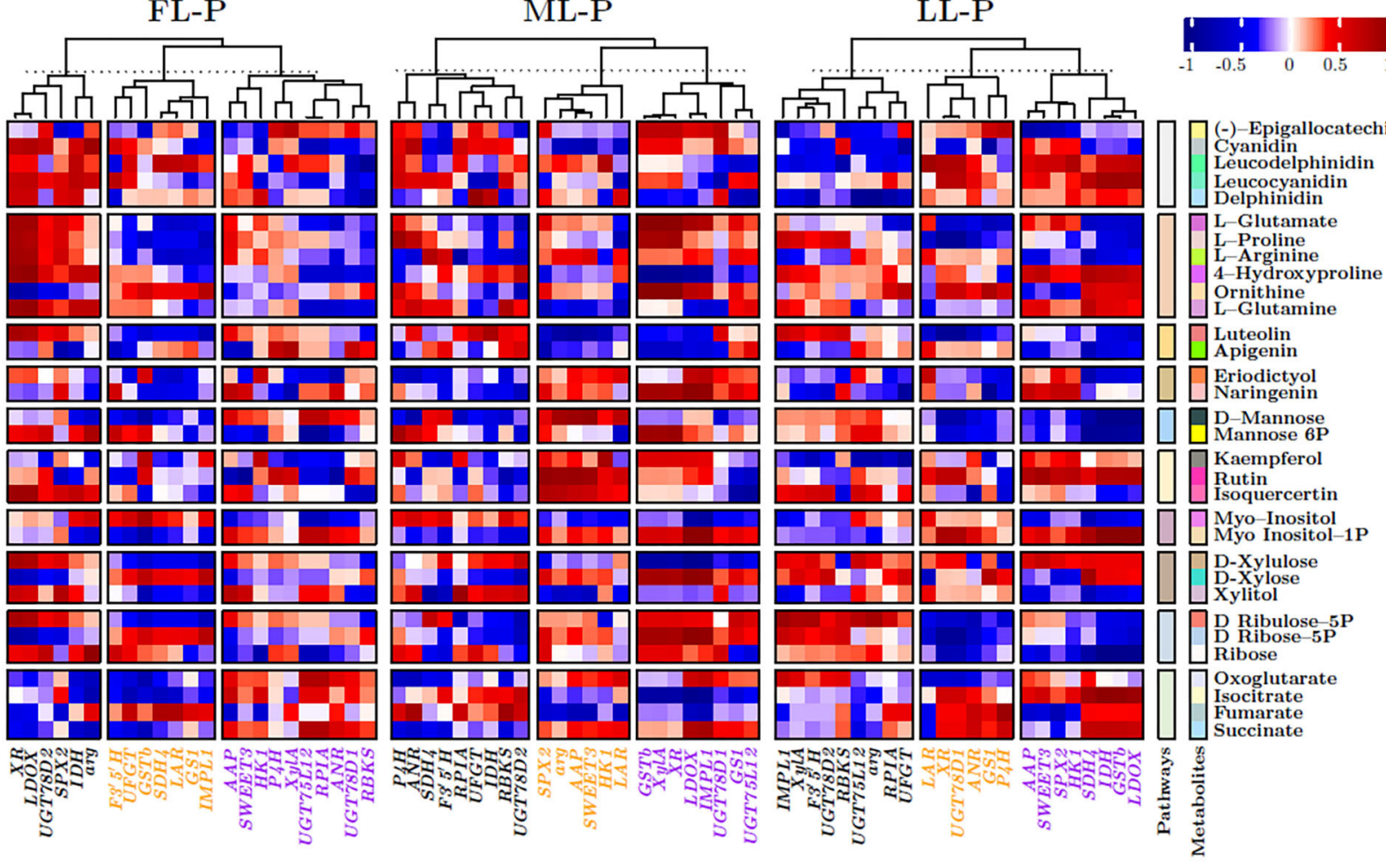

FIGURE 8 | (A,B) Heatmap correlation between P and genes under different P levels and light intensities. 


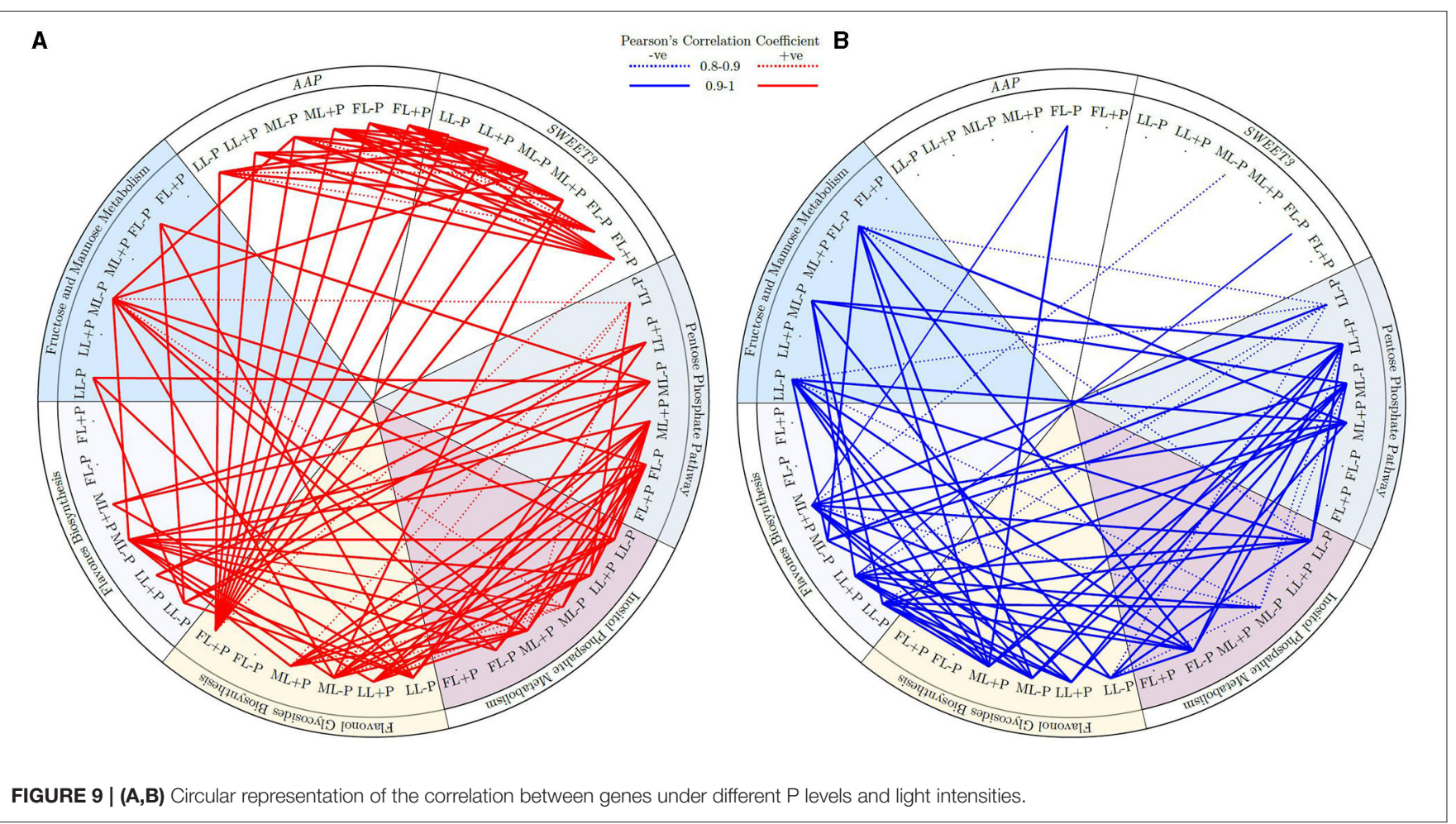

$\mathrm{P}$ was sufficient. However, the Phe concentration was reduced by further shading with $20 \%$ light intensity and also decreased by $\mathrm{P}$ deficiency. The reduction of Phe under low light and $\mathrm{P}$ deficiency indicates an irreversible effect on the physiology of the plant (Ku et al., 2010) due to its crucial roles in plant growth and defense against various types of stresses. It has been reported that asparagine transport contributes to nitrogen mobilization from source to sink organs (Oddy et al., 2020). Here, either P limitation or shading treatments reduced Asp concentration which may be associated with photorespiration and subsequently hindered nitrogen mobilization (Novitskaya et al., 2002; KC et al., 2018).

The enzymes of the TCA cycle act as a component in the electron transport chain where succinate can only be oxidized by succinate dehydrogenase (SDH). SDH activity depends on white light intensity and in our study, the expression of SDH4 decreased with shading under $\mathrm{P}$ insufficient conditions (Supplementary Figure 5). This was a response that has also been reported on Arabidopsis (Popov et al., 2010). Furthermore, the induced expression of $\mathrm{SDH} 4$ by $\mathrm{P}$ deficiency resulted in the decrease of succinate and increase of fumarate. In addition, the reduced expression of SDH4 by shading caused the accumulation of succinate and decrease of fumarate, suggesting the transcriptional regulation on $\mathrm{SDH} 4$ by light and $\mathrm{P}$. The enzyme GS1, located in the cytosol, contributes to metabolic systems via the regulation of ammonium assimilation (Kusano et al., 2020). The decrease in substrate L-glutamine and reduction in expression of GS1 negatively affect the amino acid homeostasis and plastid development in young shoots due to shading and $\mathrm{P}$ limitation. The arginine and proline metabolism plays critical roles in plant development and stress resistance that regulates polyamines independently (Majumdar et al., 2016). The response of $\mathrm{P} 4 \mathrm{H}$ expression was not consistent with the changes of proline and 4-hydroxyproline due to the diverse substrates for $\mathrm{P} 4 \mathrm{H}$ (Gorres and Raines, 2010). Arg catabolism serves to mobilize the stored nitrogen and fine-tuning the developmental and defense mechanisms against stress (Winter et al., 2015). Thus, it was assumed that the increase of Arg due to P deficiency under LL may regulate physiological and developmental processes, as well as responses to abiotic stress.

Several studies have shown that light promotes catechin synthesis and shading was effective for inhibition of the flavonoid biosynthesis (Wang et al., 2012; Li et al., 2018). Similarly, our data shows decreased catechins (ECG, EGC, and EGCG) under ML when $\mathrm{P}$ was sufficient. A compromised 3-O-glycosylation led to the repression of flavonol biosynthesis along with the inhibition of flavanol biosynthetic genes (Yin et al., 2012). The 3$\mathrm{O}$-glycosylation of flavanols is catalyzed by the UGT78D family, with UGT78D1 using UDP-rhamnose, and UGT78D2 using UDP-glucose (Yonekura-Sakakibara et al., 2008). In flavonol glycosides biosynthesis, the substrate isoquercertin was decreased due to $\mathrm{P}$ limitation combined with shading, indicating that UDP-glucose was highly consumed while UDP-rhamnose was highly utilized to form the substrate rutin when exposed to medium light during $\mathrm{P}$ deficiency in young shoots. The enzyme F3' 5 ' $\mathrm{H}$ hydroxylates the $3^{\prime}$ and 5 ' positions of dihydroflavonols (Tanaka and Brugliera, 2013; Vikhorev et al., 2019). The decrease in eriodictyol and increase in $\mathrm{F}^{\prime} 5^{\prime} \mathrm{H}$ compete for substrate naringenin during $\mathrm{P}$ deficiency in ML. Leucoanthocyanidin reductase (LARs) has been reported to promote the biosynthesis of catechin monomers and inhibit their polymerization (Wang 
et al., 2018). In contrast, our data show that catechin monomers and polymers were increased by upregulating the expression of LAR (Wang et al., 2018). This could be due to the differences between the tea plants and tobacco (Nicotiana tabacum) which were domesticated for distinct purposes. In addition, the increase in Delphinidin-3-glucoside may have been supported by UFGT which was upregulated due to P limitation and shading. Similar results have been reported previously (KC et al., 2018). The enzyme LDOX is involved in anthocyanidin and anthocyanidins biosynthesis by catalyzing the oxidation of leucoanthocyanidins to cyanidins. Our results agree with a previous report where LDOX and its synthesized cyanidins increased due to $\mathrm{P}$ limitation. Furthermore, Cyanidin 3-glucoside, involved in energy metabolism and synthesized by anthocyanidin reductase (ANR), was increased by shading.

Sensing the changes in internal nutrients is essential for plants to reprogram and adapt to fluctuating environments. For instance, the cellular P levels activate the central regulator for $\mathrm{P}$ starvation responses and P homeostasis (Wang et al., 2014). In rice, SPX2 functions as a key component in the P-sensing mechanism to control the central regulator of $\mathrm{P}$ starvationresponsive genes. Under high cellular P, OsSPX2 interacts with the central regulator $O S P H R 2$ and inhibits $P H R 2$ from binding to the motif of phosphate starvation inducible (PSI) genes. However, under low cellular $\mathrm{P}$ conditions, OsSPX2 has a low affinity to PHR2 and results in the upregulation of PSI genes, including OsSPX2. In tea plants, most of the metabolic genes detected in the present study were increased by $\mathrm{P}$ starvation; however, the transcripts of SPX 2 were decreased by P limitation, suggesting different sensing and regulating networks in tea and rice plants. It has been reported that CsSWEET3 was downregulated under cold stress and the overexpression of the SWEET gene in Arabidopsis was unable to accumulate Fructose (Yue et al., 2015). This study shows that P limitation increased the transcript level of SWEET3; however, it decreased due to light reduction. D-fructose was increased by $\mathrm{P}$ limitation under ML and decreased by shading when $\mathrm{P}$ was sufficient. Environmental signals, such as nutrient availability and light influence the expression of AAP (amino acid permeases; Ortiz-Lopez et al., 2000; Yao et al., 2020). It has been reported that AAP1 functions in the acquisition of glutamate if present in the soil at ecologically relevant concentrations (Lee et al., 2007). In this study, the linear regression and concentration measurement revealed that the increased concentration of L-glutamate in young shoots was positively correlated with the expression of $A A P$ which was upregulated by $\mathrm{P}$ limitation combined with shading. In addition, a higher concentration of L-glutamate was observed in young shoots than in mature leaves, suggesting the mobilization of Glu from source to sink. A previous study showed that GSTb had a weak affinity for epicatechin which was decreased under $\mathrm{P}$

\section{REFERENCES}

Alasalvar, C., Topal, B., Serpen, A., Bahar, B., Pelvan, E., and Gokmen, V. (2012). Flavor characteristics of seven grades of black tea produced in Turkey. J. Agri. Food Chem. 60, 6323-6332. doi: 10.1021/jf301498p limitation but its gallates were increased in young shoots (KC et al., 2018; Liu et al., 2019). This study agrees with the previous findings that epicatechin gallates were increased in young shoots due to $\mathrm{P}$ deprivation.

\section{CONCLUSION}

In conclusion, the two environmental factors light intensity and $\mathrm{P}$ supply interacted at the transcriptional and metabolic levels. The genes and metabolites involved in amino acids, carbohydrates, and flavonoid pathways were responsive to P-deficiency and shading. Different light intensities modulated some of the metabolites (e.g., Thea, EGC) differentially. Our data shows a comprehensive analysis of tea plants in response to changing light intensities coupled with $\mathrm{P}$ limitation. This information contributes to agricultural management to improve the quality of tea and maintain the yield.

\section{DATA AVAILABILITY STATEMENT}

The original contributions presented in the study are included in the article/Supplementary Material, further inquiries can be directed to the corresponding author/s.

\section{AUTHOR CONTRIBUTIONS}

JR conceived and designed the research. SK, LL, ML, and QZ performed the experiments and data interpretation. SK and LL wrote and revised the manuscript. All authors read and approved the manuscript.

\section{FUNDING}

This work was financially supported by Yunnan provincial special fund for construction of science and technology innovation base (202102AE090038), Chinese Academy of Agricultural Sciences through Agricultural Sciences Innovation Project (CAAS-ASTIP-2020-TRICAAS), China Agriculture Research System of MOF and MARA (CARS 19), Special fund for scientific research of Tea Research Institute of Chinese Academy of Agricultural Sciences (grant no.1610212021001), and Zhejiang Provincial Natural Science Foundation of China (LY21C150005).

\section{SUPPLEMENTARY MATERIAL}

The Supplementary Material for this article can be found online at: https://www.frontiersin.org/articles/10.3389/fpls.2021. 743781/full\#supplementary-material characterization of two secreted purple acid phosphatase isozymes from phosphate-starved tomato (Lycopersicon esculentum) cell cultures. Eur. J. Biochem. 269, 6278-6286. doi: 10.1046/j.1432-1033.2002.0 3347.x 
Ceasar, S. A. (2020). Regulation of low phosphate stress in plants. Plant Life Under Changing Environ. 2020, 123-156. doi: 10.1016/B978-0-12-818204-8.00007-2

Cheng, L., Tang, X., Vance, C. P., White, P. J., Zhang, F., and Shen, J. (2014). Interactions between light intensity and phosphorus nutrition affect the phosphate-mining capacity of white lupin (Lupinus albus L.). J. Exp. Bot. 65, 2995-3003. doi: 10.1093/jxb/eru135

del-Pozo, J. C., Allona, I., Rubio, V., Leyva, A., de-la-Pena, A., Aragoncillo, C., et al. (1999). A type 5 acid phosphatase gene from Arabidopsis thaliana is induced by phosphate starvation and by some other types of phosphate mobilising/oxidative stress conditions. Plant J. 19, 579-589. doi: 10.1046/j.1365-313X.1999.00562.x

Ding, Z., Jia, S., Wang, Y., Xiao, J., and Zhang, Y. (2017). Phosphate stresses affect ionome and metabolome in tea plants. Plant Physiol. Biochem. 12, 30-39. doi: 10.1016/j.plaphy.2017.09.007

Fox, T., and Comerford, N. (1990). Low-molecular-weight organic acids in selected forest soils of the southeastern USA. Soil Sci. Soc. Am. J. 54, 1139-1144. doi: 10.2136/sssaj1990.03615995005400040037x

Gillaspy, G. E. (2011). The cellular language of myo-inositol signaling. New Phytol. 192, 823-839. doi: 10.1111/j.1469-8137.2011.03939.x

Gipson, A. B., Morton, K. J., Rhee, R. J., Simo, S., Clayton, J. A., Perrett, M. E., et al. (2017). Disruptions in valine degradation affect seed development and germination in Arabidopsis. Plant J. 90, 1029-1039. doi: 10.1111/tpj.13538

Gorres, K. L., and Raines, R. T. (2010). Prolyl 4-hydroxylase. Crit. Rev. Biochem. Mol. Biol. 45, 106-124. doi: 10.3109/10409231003627991

Granot, D., Kelly, G., Stein, O., and David-Schwartz, R. (2014). Substantial roles of hexokinase and fructokinase in the effects of sugars on plant physiology and development. J. Exp. Bot. 65, 809-819. doi: 10.1093/jxb/ert400

Hammond, J. P., and White, P. J. (2008). Sucrose transport in the phloem: integrating root responses to phosphorus starvation. J. Exp. Bot. 59, 93-109 doi: $10.1093 / \mathrm{jxb} / \mathrm{erm}, 221$

KC, S., Liu, M., Zhang, Q., Fan, K., Shi, Y., Ruan, J., et al. (2018). Metabolic changes of amino acids and flavonoids in tea plants in response to inorganic phosphate limitation. Int. J. Mol. Sci. 19:3683. doi: 10.3390/ijms19113683

Kruger, N. J., and von-Schaewen, A. (2003). The oxidative pentose phosphate pathway: structure and organisation. Curr. Opin. Plant Biol. 6, 236-246. doi: $10.1016 /$ S1369-5266(03)00039-6

Ku, K. M., Choi, J. N., Kim, J., Kim, J. K., Yoo, L. G., Lee, S. J., et al. (2010). Metabolomics analysis reveals the compositional differences of shade grown tea (Camellia sinensis L.). J. Agri. Food Chem. 58, 418-426. doi: 10.1021/jf902929h

Kusano, M., Fukushima, A., Tabuchi-Kobayashi, M., Funayama, K., Kojima, S., Maruyama, K., et al. (2020). Cytosolic Glutamine Synthetase 1; 1 modulates metabolism and chloroplast development in roots. Plant Physiol. 182, 1894-1909. doi: 10.1104/pp.19.01118

Lam, H. M., Coschigano, K., Oliveira, I., Melo-Oliveira, R., and Coruzzi, G. (1996). The molecular-genetics of nitrogen assimilation into amino acids in higher plants. Ann. Rev. Plant Biol. 47, 569-593. doi: 10.1146/annurev.arplant.47.1.569

Lee, Y. H., Foster, J., Chen, J., Voll, L. M., Weber, A. P., and Tegeder, M. (2007). AAP1 transports uncharged amino acids into roots of Arabidopsis. Plant J. 50, 305-319. doi: 10.1111/j.1365-313X.2007.03045.x

Lejay, L., Wirth, J., Pervent, M., Cross, J. M., Tillard, P., and Gojon, A. (2008). Oxidative pentose phosphate pathway-dependent sugar sensing as a mechanism for regulation of root ion transporters by photosynthesis. Plant Physiol. 146, 2036-2053. doi: 10.1104/pp.107.114710

Li, H., Lin, Y., Chen, X., Bai, Y., Wang, C., Xu, X., et al. (2018). Effects of blue light on flavonoid accumulation linked to the expression of miR393, miR394 and miR395 in longan embryogenic calli. PLoS ONE 13:e0191444. doi: 10.1371/journal.pone.0191444

Liang, C., Tian, J., Lam, H. M., Lim, B. L., Yan, X., and Liao, H. (2010). Biochemical and molecular characterization of PvPAP3, a novel purple acid phosphatase isolated from common bean enhancing extracellular ATP utilization. Plant Physiol. 152, 854-865. doi: 10.1104/pp.109.147918

Liao, H., Yan, X. L., Rubio, G., Beebe, S. E., Blair, M. W., and Lynch, J. P. (2004). Genetic mapping of basal root gravitropism and phosphorus acquisition efficiency in common bean. Funct. Plant Biol. 10, 959-997. doi: 10.1071/FP03255

Ligaba, A., Yamaguchi, M., Shen, H., Sasaki, T., Yamamoto, Y., and Matsumoto, H. (2004). Phosphorus deficiency enhances plasma membrane $\mathrm{H}^{+}$-ATPase activity and citrate exudation in greater purple lupin (Lupinus pilosus). Funct. Plant Biol. 31, 1075-1083. doi: 10.1071/FP04091

Lin, Z. H., Qi, Y. P., Chen, R. B., Zhang, F. Z., and Chen, L., S. (2012). Effects of phosphorus supply on the quality of green tea. Food Chem. 130, 908-914. doi: 10.1016/j.foodchem.2011.08.008

Liu, Y., Jiang, H., Zhao, Y., Li, X., Dai, X., Zhuang, J., et al. (2019). Three Camellia sinensis glutathione S-transferases are involved in the storage of anthocyanins, flavonols, and proanthocyanidins. Planta 250, 1163-1175. doi: 10.1007/s00425-019-03206-2

Majumdar, R., Barchi, B., Turlapati, S. A., Gagne, M., Minocha, R., Long, S., et al. (2016). Glutamate, ornithine, arginine, proline, and polyamine metabolic interactions: the pathway is regulated at the post-transcriptional level. Front. Plant Sci. 16, 7-78. doi: 10.3389/fpls.2016.00078

Miyauchi, S., Yuki, T., Fuji, H., Kojima, K., Yonetani, T., Tomio, A., et al. (2014). High-quality green tea leaf production by artificial cultivation under growth chamber conditions considering amino acids profile. J. Biosci. Bioeng. 118, 710-715. doi: 10.1016/j.jbiosc.2014.05.008

Motalebifard, R., Najafi, N., Oustan, S., Nyshabouri, M. R., and Valizadeh, M. (2013). The combined effects of phosphorus and zinc on evapotranspiration, leaf water potential, water use efficiency and tuber attributes of potato under water deficit conditions. Sci. Horticult. 162, 31-38. doi: 10.1016/j.scienta.2013.07.043

Müller, R., Nilsson, L., Nielsen, L. K., and Hamborg-Nielsen, T. (2005). Interaction between phosphate starvation signalling and hexokinaseindependent sugar sensing in Arabidopsis leaves. Physiol. Plant. 124, 81-89. doi: 10.1111/j.1399-3054.2005.00496.x

Novitskaya, L., Trevanion, S. J., Driscoll, S., Foyer, C. H., and Noctor, G. (2002). How does photorespiration modulate leaf amino acid contents? A dual approach through modelling and metabolite analysis. Plant Cell Environ. 25, 821-835. doi: 10.1046/j.1365-3040.2002.00866.x

Oddy, J., Raffan, S., Wilkinson, M. D., Elmore, J. S., and Halford, N. G. (2020). Stress, nutrients and genotype: understanding and managing asparagine accumulation in wheat grain. CABI Agri. Biosci. 1, 1-4. doi: 10.1186/s43170-020-00010-x

Ortiz-Lopez, A., Chang, H. C., and Bush, D. R. (2000). Amino acid transporters in plants. Biochim. Biophys. Acta 1465, 275-228. doi: 10.1016/S0005-2736(00)00144-9

Pant, B. D., Pant, P., Erban, A., Huhman, D., Kopka, J., and Scheible, W. R. (2015). Identification of primary and secondary metabolites with phosphorus statusdependent abundance in Arabidopsis, and of the transcription factor PHR1 as a major regulator of metabolic changes during phosphorus limitation. Plant Cell Environ. 38, 172-187. doi: 10.1111/pce.12378

Popov, V. N., Eprintsev, A. T., Fedorin, D. N., and Igamberdiev, A. U. (2010). Succinate dehydrogenase in Arabidopsis thaliana is regulated by light via phytochrome A. FEBS Lett. 584, 199-202. doi: 10.1016/j.febslet.2009.11.057

Quehenberger, J., Reichenbach, T., Baumann, N., Rettenbacher, L., Divne, C., and Spadiut, O. (2019). Kinetics and predicted structure of a novel xylose reductase from Chaetomium thermophilum. Int. J. Mol. Sci. 20:185. doi: $10.3390 /$ ijms 20010185

Robinson, W. D., Park, J., Tran, H. T., Del-Vecchio, H. A., Ying, S., Zins, J. L., et al. (2012). The secreted purple acid phosphatase isozymes AtPAP12 and AtPAP26 play a pivotal role in extracellular phosphate-scavenging by Arabidopsis thaliana. J. Exp. Bot. 63, 6531-6542. doi: 10.1093/jxb/e rs309

Ruan, J., Ma, L., and Yang, Y. (2012). Magnesium nutrition on accumulation and transport of amino acids in tea plants. J. Sci. Food Agri. 92, 1375-1383. doi: 10.1002/jsfa.4709

Salahinejad, M., and Aflaki, F. (2010). Toxic and essential mineral elements content of black tea leaves and their tea infusions consumed in Iran. Biol. Trace Elemement Res. 134, 109-117. doi: 10.1007/s12011-009-8449-z

Schlüter, U., Colmsee, C., Scholz, U., Bräutigam, A., Weber, A. P., Zellerhoff, N., et al. (2013). Adaptation of maize source leaf metabolism to stress related disturbances in carbon, nitrogen and phosphorus balance. BMC Genom. 14, 1-25. doi: 10.1186/1471-2164-14-442

Shen, J. B., Yuan, L. X., Zhang, J. L., Li, H. G., Bai, Z. H., Chen, X. P., et al. (2011). Phosphorus dynamics: from soil to plant. Plant Physiol. 156, 997-1005. doi: $10.1104 /$ pp.111.175232 
Singh, H. R., Deka, M., and Das, S. (2015). Enhanced resistance to blister blight in transgenic tea (Camellia sinensis [L.], O. Kuntze) by overexpression of class I chitinase gene from potato (Solanum tuberosum). Funct. Integrative Genomi. 15, 461-448. doi: 10.1007/s10142-015-0436-1

Stewart, A., Chapman, W., Jenkins, G., Graham, I., Martin, T., and Crozier, A. (2001). The effect of nitrogen and phosphorus deficiency on flavonol accumulation in plant tissues. Plant Cell Environ. 24, 1189-1197 doi: 10.1046/j.1365-3040.2001.00768.x

Stilo, F., Tredici, G., Bicchi, C., Robbat, A., Morimoto, J., and Cordero, C. (2020). Climate and processing effects on tea (Camellia sinensis, L. Kuntze) metabolome: accurate profiling and fingerprinting by comprehensive twodimensional gas chromatography/time-of-flight mass spectrometry. Molecules 25:2447. doi: 10.3390/molecules25102447

Ström, L., Owen, A. G., Godbold, D. L., and Jones, D. L. (2005). Organic acid behaviour in a calcareous soil implications for rhizosphere nutrient cycling. Soil Biol. Biochem. 37, 2046-2054. doi: 10.1016/j.soilbio.2005.03.009

Taghipour, M., and Jalali, M. (2013). Effect of low-molecular-weight organic acids on kinetics release and fractionation of phosphorus in some calcareous soils of western Iran. Environ. Monitor. Assess. 185, 5471-5482. doi: 10.1007/s10661-012-2960-y

Tanaka, Y., and Brugliera, F. (2013). Flower colour and cytochromes P45. Philos. Trans. Royal Soc. B Biol. Sci. 368:20120432. doi: 10.1098/rstb.2012.0432

Taylor, M. D., Nelson, P. V., and Frantz, J. M. (2008). Substrate acidification by geranium: light effects and phosphorus uptake. J. Am. Soc. Horticult. Sci. 133, 515-552. doi: 10.21273/JASHS.133.4.515

Tian, J., Wang, X., Tong, Y., Chen, X., and Liao, H. (2012). Bioengineering and management for efficient phosphorus utilization in crops and pastures. Curr. Opin. Biotechnol. 23, 866-871. doi: 10.1016/j.copbio.2012.03.002

Timm, S., Florian, A., Wittmi,ß M., Jahnke, K., Hagemann, M., Fernie, A. R., et al. (2013). Serine acts as a metabolic signal for the transcriptional control of photorespiration-related genes in Arabidopsis. Plant Physiol. 162, 379-389. doi: $10.1104 /$ pp.113.215970

Vikhorev, A. V., Strygina, K. V., and Khlestkina, E. K. (2019). Duplicated flavonoid 3'-hydroxylase and flavonoid 3', 5'-hydroxylase genes in barley genome. PeerJ 7:e6266. doi: $10.7717 /$ peerj.6266

Wang, C., Ying, S., Huang, H., Li, K., Wu, P., and Shou, H. (2009). Involvement of OsSPX1 in phosphate homeostasis in rice. Plant J. 57, 895-904. doi: 10.1111/j.1365-313X.2008.03734.x

Wang, L., Zhou, T., Cheng, B., Du, Y., Qin, S., Gao, Y., et al. (2020). Variable light condition improves root distribution shallowness and $\mathrm{p}$ uptake of soybean in maize/soybean relay strip intercropping system. Plants 9:1204. doi: $10.3390 /$ plants 9091204

Wang, P., Zhang, L., Jiang, X., Dai, X., Xu, L., Li, T., et al. (2018). Evolutionary and functional characterization of leucoanthocyanidin reductases from Camellia sinensis. Planta 247, 139-154. doi: 10.1007/s00425-017-2771-Z

Wang, Y., Gao, L., Shan, Y., Liu, Y., Tian, Y., and Xia, T. (2012). Influence of shade on flavonoid biosynthesis in tea [Camellia sinensis (L.) O. Kuntze]. Sci. Horticult. 141, 7-16. doi: 10.1016/j.scienta.2012.04.013

Wang, Z., Ruan, W., Shi, J., Zhang, L., Xiang, D., Yang, C., et al. (2014). Rice SPX1 and SPX2 inhibit phosphate starvation responses through interacting with PHR2 in a phosphate-dependent manner. Proc. Natl. Acad. Sci. U. S. A. 111, 14953-14958. doi: 10.1073/pnas.1404680111

Winter, G., Todd, C. D., Trovato, M., Forlani, G., and Funck, D. (2015). Physiological implications of arginine metabolism in plants. Front. Plant Sci. 6:534. doi: 10.3389/fpls.2015.00534
Xiong, L., Li, J., Li, Y., Yuan, L., Liu, S., Huang, J., et al. (2013). Dynamic changes in catechin levels and catechin biosynthesis-related gene expression in albino tea plants (Camellia sinensis L.). Plant Physiol. Biochem. 71, 132-143. doi: 10.1016/j.plaphy.2013.06.019

Yang, Z., Kobayashi, E., Katsuno, T., Asanuma, T., Fujimori, T., Ishikawa, T., et al. (2012). Characterisation of volatile and non-volatile metabolites in etiolated leaves of tea (Camellia sinensis) plants in the dark. Food Chem. 135, 2268-2276. doi: 10.1016/j.foodchem.2012.07.066

Yao, X., Nie, J., Bai, R., and Sui, X. (2020). Amino acid transporters in plants: identification and function. Plants 9:972. doi: 10.3390/plants 9080972

Yin, R., Messner, B., Faus-Kessler, T., Hoffmann, T., Schwab, W., Hajirezaei, M. R., et al. (2012). Feedback inhibition of the general phenylpropanoid and flavonol biosynthetic pathways upon a compromised flavonol-3-O-glycosylation. $J$. Exp. Bot. 63, 2465-2478. doi: 10.1093/jxb/err416

Yonekura-Sakakibara, K., Tohge, T., Matsuda, F., Nakabayashi, R., Takayama, H., Niida, R., et al. (2008). Comprehensive flavonol profiling and transcriptome coexpression analysis leading to decoding gene-metabolite correlations in Arabidopsis. Plant Cell 20, 2160-2176. doi: 10.1105/tpc.108.058040

Yue, C., Cao, H. L., Wang, L., Zhou, Y. H., Huang, Y. T., Hao, X. Y., et al. (2015). Effects of cold acclimation on sugar metabolism and sugar-related gene expression in tea plant during the winter season. Plant Mol. Biol. 88, 591-608. doi: $10.1007 / \mathrm{s} 11103-015-0345-7$

Zhang, Q., Liu, M., Mumm, R., Vos, R. C. H., and Ruan, J. (2021). Metabolomics reveals the within-plant spatial effects of shading on tea plants. Tree Physiol. 41 317-333. doi: 10.1093/treephys/tpaa127

Zhang, R. G., Andersson, C. E., Savchenko, A., Skarina, T., Evdokimova, E., Beasley, S., et al. (2003). Structure of Escherichia coli ribose-5-phosphate isomerase: a ubiquitous enzyme of the pentose phosphate pathway and the Calvin cycle. Structure 11, 31-42. doi: 10.1016/S0969-2126(02)00933-4

Zhou, J., Jiao, F. C., Wu, Z., Li, Y., Wang, X., He, X., et al. (2008). OsPHR2 is involved in phosphate-starvation signaling and excessive phosphate accumulation in shoots of plants. Plant Physiol. 146, 1673-1686 doi: 10.1104/pp.107.111443

Zhou, T., Wang, L., Sun, X., Wang, X., Chen, Y., Rengel, Z., et al. (2020). Light intensity influence maize adaptation to low $\mathrm{P}$ stress by altering root morphology. Plant Soil 447, 183-197. doi: 10.1007/s11104-019-04259-8

Conflict of Interest: The authors declare that the research was conducted in the absence of any commercial or financial relationships that could be construed as a potential conflict of interest.

Publisher's Note: All claims expressed in this article are solely those of the authors and do not necessarily represent those of their affiliated organizations, or those of the publisher, the editors and the reviewers. Any product that may be evaluated in this article, or claim that may be made by its manufacturer, is not guaranteed or endorsed by the publisher.

Copyright (C) 2021 KC, Long, Liu, Zhang and Ruan. This is an open-access article distributed under the terms of the Creative Commons Attribution License (CC BY). The use, distribution or reproduction in other forums is permitted, provided the original author(s) and the copyright owner(s) are credited and that the original publication in this journal is cited, in accordance with accepted academic practice. No use, distribution or reproduction is permitted which does not comply with these terms. 


\section{GLOSSARY}

HXK1, hexokinase 1; RPIA, ribose 5-phosphate isomerase A; GS1, glutamate synthetase 1; $\mathrm{P} 4 \mathrm{H}$, prolyl 4-hydroxylase; Thea, Theanine; GC $\times$ GC-TOF/MS, Two-dimensional Gas Chromatography coupled to Time-of-Flight Mass Spectrometry; UPLC-Q-TOF/MS, Ultra-Performance Liquid Chromatography-Quadrupole-Time of Flight Mass Spectrometry; ICP-AES, Inductively Coupled PlasmaAtomic Emission Spectroscopy; qRT-PCR, quantitative Real Time-Polymerase Chain Reaction; GAPDH, glyceraldehyde 3phosphate dehydrogenase; PCA, Principal Component Analysis; PLS-DA, Partial Least Squares-Discriminant Analysis; Glu, Glutamate; Pro, Proline; Arg, Arginine; EGC, Epigallocatechin; GC, Gallocatechin; GCG, Gallocatechin gallate; GA, Gallic acid; SPX2, Pi transport, stress, sensing, and signaling; SWEET3, bidirectional sugar transporter; AAP, amino acid permeases; GSTb, glutathione S-transferase b; RBKS, ribokinase synthase; IMPL1, inositol-phosphate phosphatase 1; XylA, xylose isomerase; IDH, isocitrate dehydrogenase; $L D O X$, leucoanthocyanidin dioxygenase; UFGT, anthocyanidin 3-O-glucosyltransferase; UGT8D1, flavonol-3-O-glucoside L-rhamnosyltransferase; UGT78D2, flavonol-3-O-glycosyltransferase; UGT75L12, glucosyltransferases; FMM, Fructose and Mannose Metabolism; TCA, Tricarboxylic acid cycle; SDH, Succinate dehydrogenase; F3'5'H, flavonoid 3',5'hydroxylase; ANR, anthocyanidin reductase; PHR, phosphate starvation response. 